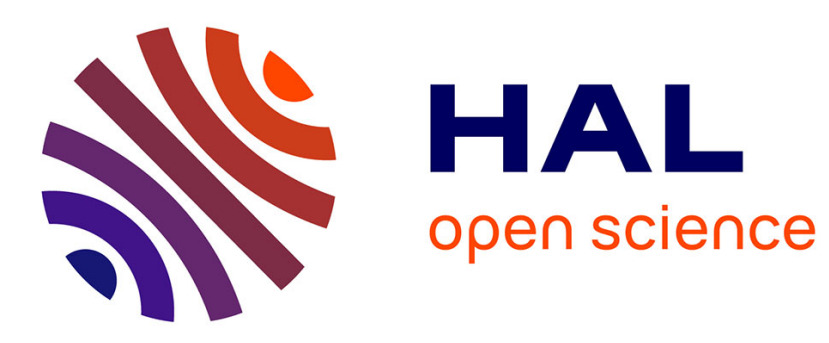

\title{
On a uniformly valid analytical rectilinear cascade response function
}

Hélène Posson, Michel Roger, Stéphane Moreau

\section{To cite this version:}

Hélène Posson, Michel Roger, Stéphane Moreau. On a uniformly valid analytical rectilinear cascade response function. Journal of Fluid Mechanics, 2010, 663, pp.22-52. 10.1017/S0022112010003368 . hal-00566055

\section{HAL Id: hal-00566055 https://hal.science/hal-00566055}

Submitted on 7 Jun 2012

HAL is a multi-disciplinary open access archive for the deposit and dissemination of scientific research documents, whether they are published or not. The documents may come from teaching and research institutions in France or abroad, or from public or private research centers.
L'archive ouverte pluridisciplinaire HAL, est destinée au dépôt et à la diffusion de documents scientifiques de niveau recherche, publiés ou non, émanant des établissements d'enseignement et de recherche français ou étrangers, des laboratoires publics ou privés. 


\title{
On a uniformly valid analytical rectilinear cascade response function
}

\author{
HELENE POSSON ${ }^{1} \dagger$, M. ROGER AND S. MOREAU ${ }^{2}$ \\ ${ }^{1}$ Laboratoire de Mécanique des Fluides et Acoustique, École Centrale de Lyon, \\ 69134 Écully CEDEX, France \\ ${ }^{2}$ G.A.U.S., Mechanical Engineering Department, Université de Sherbrooke, Sherbrooke, QC, \\ Canada J1K 2R1
}

(Received 17 June 2009; revised 17 June 2010; accepted 21 June 2010; first published online 27 September 2010)

This paper extends an existing analytical model of the aeroacoustic response of a rectilinear cascade of flat-plate blades to three-dimensional incident vortical gusts, by providing closed-form expressions for the acoustic field inside the inter-blade channels, as well as for the pressure jump over the blades in subsonic flows. The extended formulation is dedicated to future implementation in a fan-broadbandnoise-prediction tool. The intended applications include the modern turbofan engines, for which analytical modelling is believed to be a good alternative to more expensive numerical techniques. The initial model taken as a reference is based on the WienerHopf technique. An analytical solution valid over the whole space is first derived by making an extensive use of the residue theorem. The accuracy of the model is shown by comparing with numerical predictions of benchmark configurations available in the literature. This full exact solution could be used as a reference for future assessment of numerical solvers, of linearized Euler equations for instance, in rectilinear or narrow-annulus configurations. In addition, the pressure jump is a key piece of information because it can be used as a source term in an acoustic analogy when the rectilinear-cascade model is applied to three-dimensional blade rows by resorting to a strip-theory approach. When used as such in a true rectilinear-cascade configuration, it reproduces the exact radiated field that can be derived directly. The solution is also compared to a classical single-airfoil formulation to highlight the cascade effect. This effect is found important when the blades of the cascade overlap significantly, but the cascade solution tends to the single-airfoil one as the overlap goes to zero. This suggests that both models can be used as the continuation of each other if needed.

Key words: aeroacoustics

\section{Introduction}

The design of modern turbofan engines involves larger diameters and higher bypass ratios than previous technologies, for improved aircraft performance at lower nominal rotation speed. The noise from the jet is reduced as a result of the lower exhaust velocity of burnt gases. As a result, the fan noise associated with sources distributed over the rotor blades and the outlet guide vanes has turned out to be a major

$\dagger$ Present address: G.A.U.S., Mechanical Engineering Department, Université de Sherbrooke, Sherbrooke, QC, Canada J1K 2R1. Email address for correspondence: helene.posson@gmail.com 
contribution to the total noise, especially during approach and landing of an aircraft. Even though liners are currently integrated in engine duct walls to attenuate fan noise, their benefit is partly limited by the relatively shorter duct length needed for weight and drag constraints in large-bypass-ratio architectures. Therefore, noise reduction at the source remains a concern for engine and/or aircraft manufacturers. In this context, accurate prediction schemes, including the relative and absolute effects of the geometrical parameters of the fan, become essential to a quieter design.

New technological options involve lower fan-tip speed, reduced number of blades, and selected blade and vane counts. This ensures tonal noise control and shifts the tone frequencies to lower values associated with weaker loudness. As a result, the broadband noise contribution becomes relatively more significant, and dedicated acoustic predictions are a crucial step to be included in the design cycles, as early and as accurately as possible. Broadband noise is essentially generated because of the interaction of turbulence with the blade and vane surfaces. This includes fan rotor noise due to ingestion of turbulence either in the inner flow or in the casing and spinner boundary layers, rotor trailing-edge noise and stator noise due to the impingement of the rotor wakes. This study addresses broadband-noise contributions resulting from the impingement of incident turbulence on a blade row. Currently, the prediction of fan broadband noise over the extended frequency range of aeronautical applications, typically between $50 \mathrm{~Hz}$ and $8 \mathrm{kHz}$ according to effective perceived noise level standards, is still a daunting task. Apart from the Reynolds-number and Mach-number issues, and from the complex geometry, the Helmholtz numbers $k R_{T}$ or $k c$ based on either the duct outer diameter $R_{T}$ or the blade chord $c$ typically range up to 100 and 30 , respectively. The acoustic wavenumber is defined as $k=\omega / c_{0}$, with $c_{0}$ being the speed of sound and $\omega$ the angular frequency. This makes the turboengine fan a very challenging case going from relatively low to very high frequencies. Computational aeroacoustics or computational fluid dynamics simulations dealing with so different scales are simply not realistic in the short term for industrial purposes. In recent years, linearized Euler codes have been developed to solve twodimensional (e.g. Hall \& Verdon 1991; Fang \& Atassi 1993) and progressively fully three-dimensional annular-cascade problems with mean swirling flow (e.g. Golubev \& Atassi 2000; Atassi et al. 2004) and loading, and have been successfully applied to broadband computations (e.g. Atassi \& Vinogradov 2005, 2007; Atassi \& Logue 2008). These methods developed in a research context are intermediate between direct numerical simulations or large-eddy simulations and analytical or semi-numerical models. Even though they should be used in industry in the near future, their simulation time is still too long to fit into an actual optimization loop. This is why a full analytical approach is preferred here. Fast-running analytical models are more appropriate for the parametric studies required during the preliminary design of a fan. The price to pay is the possible inaccuracy inherent in the necessary simplifications made to get closed-form solutions. Typically, a blade is locally represented by a flat plate with zero thickness and the relative mean flow over the blade rows is assumed uniform and parallel to the chord line. In fact, as pointed out by Evers \& Peake (2002), the real shape of blades with small camber and thickness has an important effect on the interferences producing the tonal noise from periodic wake interactions. In contrast, a blade can be reasonably represented by a flat plate with zero thickness for a pure-broadband-noise evaluation, taking advantage of the smoothing effect of a statistical description. Another point is the mathematical complexity of prediction schemes based on a cascade response function when compared to approaches resorting to single-airfoil solutions. But even though successful predictions have been achieved 
in the past based on single-airfoil response functions for instance in the case of open rotors with four blades (e.g. Paterson \& Amiet 1979), several authors (Goldstein 1976, Atassi \& Hamad 1981 and Peake 2004, for instance) recognized the need for a proper account of the cascade effect, understood as the influence of neighbouring blades on the response of a single one, or as an ensemble behaviour somewhat similar to the diffraction of electromagnetic waves by optic gratings. This effect is already expected intuitively, since nothing can be seen across a fan rotor due to the blade overlap, except close to the hub. Therefore, sound waves cannot propagate through the rotor without being affected significantly. More generally, the cascade effect occurs on both the sound generation by a blade row and the sound transmission through a blade row. This motivated the efforts made here to derive a uniformly valid solution extending the previous analysis proposed by Glegg (1999).

The first two-dimensional rectilinear-cascade semi-numerical and analytical models were mostly developed in the 1970s. Considering a semi-numerical approach, Kaji \& Okazaki $(1970 b)$ proposed a singularity method based on the acceleration potential to treat the sound transmission through a blade row and then the sound generation from rotor-stator interaction (Kaji \& Okazaki 1970a). Whitehead (1972) derived a solution, from a lifting-surface method, providing the forces on the blades, the vortical field, the acoustic field scattered upstream and downstream of a blade row for incident vortical and acoustic gusts or bending and flutter excitation. Smith (1973) addressed the same problem with a lifting-surface method based on a distribution of bound vortices. Whitehead (1987) later implemented this method in the code LINSUB (i.e. linearized subsonic unsteady flow in cascade), which is currently used by many researchers. Goldstein (Goldstein 1976, chapter 5) proposed a solution to account for three-dimensional gusts and duct walls in a rectilinear configuration. Atassi \& Hamad (1981) used this approach to treat the interaction of wakes and secondary flows with a cascade. Their work is the basis of the code LINC (i.e. linear cascade). In addition, they derived similarity rules for three-dimensional gusts impinging on a rectilinear cascade, as established by Graham (1970) for airfoils. All these methods require numerically solving an integral equation resorting to a collocation technique in most cases.

Alternatively, models based on the Wiener-Hopf technique lead to explicit solutions. Mani \& Horvay (1970) derived a zeroth-order solution for the sound transmission through a blade row, neglecting the interaction between leading and trailing edges. Koch (1971) later introduced the backscattering necessary to couple both edges. But the resulting full formulation obtained with a finite Wiener-Hopf technique was still time-consuming and hard to apply, till Peake's new analysis of Koch's problem for the noise generation provided a quick evaluation of the unsteady loading (Peake 1993). This exact solution is obtained by matching the scattering at the leading edge and the scattering at the trailing edge with inter-blade channel modes. Peake (1992) also proposed a high-frequency approximation, which accounts for the first two terms in Landahl's approach (Landahl 1961). This solution, being valid only when radiated modes and duct modes are well cut-on, has been extended to a uniformly valid solution by Peake \& Kerschen (1995). Majumdar \& Peake (1996) extended Peake's model (Peake 1993) to a three-dimensional gust to account for spanwise variation of the incident distortion. This refinement can be important when studying both the rotor-stator interaction, as shown by Ravindranath \& Lakshminarayana (1980) and Ganz et al. (1998), or the ingestion of atmospheric turbulence by a rotor (e.g. Hanson 1974). The full exact solution for a three-dimensional gust may be derived in the same way as in the two-dimensional gust problem (Peake 1993). Yet, the authors 
addressed only high-frequency gusts for which the cascade trailing-edge effects can be neglected. Finally, Glegg (1999) applied the Wiener-Hopf technique to get the exact solution for the acoustic field radiated from a cascade, taking advantage of the fact that determining the unsteady loadings on the blades is no longer necessary to derive the acoustic potential outside the cascade. Furthermore, the model was easily adapted to infer the cascade response for three-dimensional gusts and swept blades.

In addition to all approaches resorting to a rectilinear cascade, Namba (1987) and Schulten (1982) developed semi-numerical singularity methods for a ducted threedimensional annular cascade. This included a rotor or a zero-stagger stator and then possibly swept blades (Kodama \& Namba 1990; Schulten 1997). These methods account for the first three-dimensional annular effects. But the zero-stagger restriction discards mean swirling flow effects and radial variations of the stagger. The swirl was introduced, numerically, in the three-dimensional annular problem by Golubev \& Atassi (2000) and Atassi et al. (2004) with a code solving the three-dimensional linearized Euler equations. These investigations point out many three-dimensional issues such as the complex geometry, the spanwise non-uniformity of the excitation and mean flow changes on both sides of a cascade.

This paper, based on Glegg's analytical approach, i.e. Glegg (1999), re-addresses the problem of the sound generated as turbulent gusts interact with a blade row. Some extensions are made to the original formulation. Whereas Glegg's analysis focused on the waves outside the blade row, a unified analytical solution is derived, which is valid over the whole space, including the inter-blade channels for all values of frequency. This is achieved by an intensive use of the residue theorem for complexvariable functions and provides a formula for the distributed unsteady lift over a blade. This exact formulation is equivalent to that of Peake (1993) when a twodimensional gust is considered, and extends the results to three-dimensional gusts. Writing down formulae for the unsteady lift is motivated by the future implementation in a turbulence-interaction noise model for a three-dimensional annular blade row, based on a strip-theory approach within the framework of an acoustic analogy, as detailed by Posson, Moreau \& Roger (2010). Furthermore, the full exact solution will possibly serve as a reference for future comparisons to assess numerical methods and simulations, of linearized Euler equations for instance, in rectilinear-cascade or narrow-annulus configurations.

The analytical formulation is first described in $\S 2$ where the extended equations are derived. The method is detailed only for one part of the potential field inside the inter-blade channel, and guidelines are given for the complementary derivations, for the sake of conciseness. The full solution is explicitly given in Appendix A for the sound generation configuration, both in the general case and in the particular case of a gust impinging on a zero-stagger cascade in phase on all blades. The validation of the extensions proposed for the three-dimensional rectilinear-cascade model is addressed in $\S 3$ for the unsteady loading on the blades and in $\S 4$ for the pressure field inside and outside the inter-blade channel. The unsteady loading on the blades is also used as equivalent dipole source distribution in the usual sense of an acoustic analogy to predict the radiated field in $\S 5$. The synthesized radiated field is then compared with the one directly produced by the model. Finally, to go beyond the cascade effect, the cascade solution is compared to Sears' isolated-airfoil incompressible response function in $\$ 3.2$, and to Amiet's isolated-airfoil response function with backscattering (Amiet 1975) both for the unsteady blade loading and for the radiated field in $\S \S 3.2$ and 5.2, respectively. 
(a)

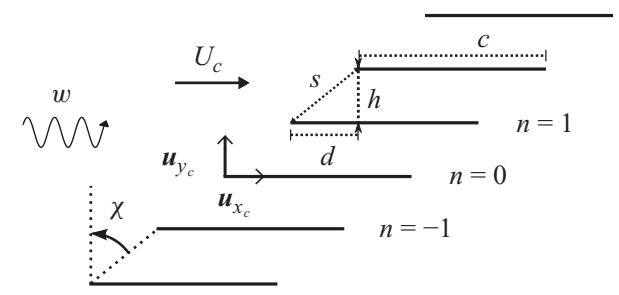

(b)
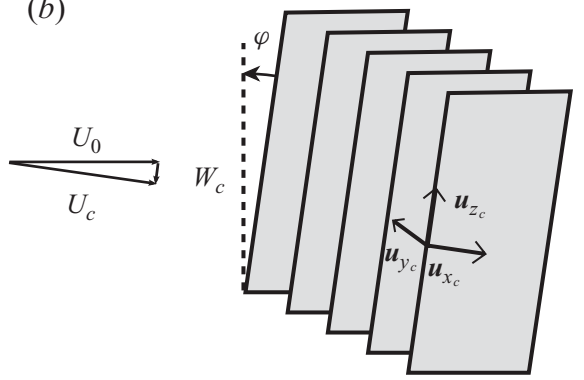

FIGURE 1. Geometry of the rectilinear-cascade model and incident velocity gust: $(a)$ side view; (b) three-dimensional view. The spanwise velocity component $W_{c}$ is non-zero only for swept blades. The blades are numbered from $n=-\infty$ to $n=+\infty$.

\section{Analytical formulation}

This section is aimed at deriving analytical expressions for the acoustic field in the inter-blade channels and the unsteady blade loading. This study is based on Glegg's analysis of cascade response functions (Glegg 1999). The model geometry and reference frames are plotted in figure 1. The blade row is assumed an equivalent rectilinear cascade of infinite spanwise extent. The blades are rigid flat plates of finite chord $c$ and zero thickness, possibly staggered with an angle $\chi$. The normal distance between successive blades is denoted by $h$. The mean flow of speed $U_{0}$ is parallel to the blade surface, which corresponds to a zero angle of attack. The sweep angle $\varphi$ splits the incident velocity into a spanwise mean flow component $W_{c}$, and a chordwise component $U_{c}$. The Cartesian coordinates $\left(x_{c}, y_{c}, z_{c}\right)$ in the cascade reference frame are defined in the chordwise $\boldsymbol{u}_{x_{c}}$, normal $\boldsymbol{u}_{y_{c}}$ and spanwise $\boldsymbol{u}_{z_{c}}$ directions, respectively. The incident wave pattern here is a vortical gust convected downstream by the mean flow. The upwash gust consists of a harmonic wave of amplitude $w_{0}$ normal to the blade at angular frequency $\omega_{e x}$ with wavenumbers $\left(k_{x_{c 0}}, k_{y_{c 0}}, k_{z_{c 0}}\right)$ in the cascade reference frame, and hence is defined by

$$
\boldsymbol{w} \cdot \boldsymbol{u}_{y_{c}}=w_{0} \exp \left(-\mathrm{i} \omega_{e x} t+\mathrm{i} k_{x_{c 0}} x_{c}+\mathrm{i} k_{y_{c 0}} y_{c}+\mathrm{i} k_{z_{c 0}} z_{c}\right)
$$

It should be stressed that the stagger distance $d$ cannot be larger than the chord length $c$, i.e. the adjacent blades must have a non-zero overlap. This condition is necessary when solving the iterative Wiener-Hopf equations successively applied to the leading-edge and trailing-edge diffraction problems. Both edges are coupled by the guided waves in the overlapping region of the inter-blades channels. Some extensions are proposed here to get analytical expressions for all quantities of interest everywhere inside and outside the blade row.

Since all assumptions are kept the same as in Glegg's analysis, the starting point is the expression for the velocity potential of the scattered field $\widehat{\phi}$ in the strip $0 \leqslant y_{c}<h$, reproduced here from (31) of Glegg (1999):

$$
\begin{aligned}
& \widehat{\phi}\left(x_{c}, y_{c}, z_{c}, t\right)=\frac{1}{2} \int_{-\infty}^{\infty} D\left(k_{x_{c}}\right) \exp \left(-\mathrm{i} k_{x_{c}} x_{c}-\mathrm{i} \omega_{e x} t+\mathrm{i} k_{z_{c}} z_{c}\right) \\
& \quad \times\left\{\frac{\mathrm{e}^{\mathrm{i} \zeta y_{c}}}{1-\exp \left(\mathrm{i} \zeta h-\mathrm{i} k_{x_{c}} d-\mathrm{i} \sigma\right)}+\frac{\mathrm{e}}{1-\exp \left(-\mathrm{i} \zeta h-\mathrm{i} k_{x_{c}} d-\mathrm{i} \sigma\right)}\right\} \mathrm{d} k_{x_{c}} .
\end{aligned}
$$

The expression of $\widehat{\phi}$ is in fact the opposite of the one given in the reference equation (31) due to a misprint (omission of a minus sign in (11) after the integration over $y_{c}$ 
in (10) of the reference paper). The present expression involves the Fourier transform of the velocity-potential jump across the blade $D$. The latter is the sum of four terms $D^{(j)}, j=1,2,3,4$, given in Appendix A.1 from Glegg's results. $D^{(1)}$ stands for the direct scattering of the incident gust by the leading edge, with the cascade being assumed to be a row of semi-infinite plates extending to infinity downstream. $D^{(2)}$ is the trailing-edge backscattering which accounts for the Kutta condition at the trailing edge and deals with a row of semi-infinite plates extending to infinity upstream. Since the resulting solution using $D^{(1)}+D^{(2)}$ no longer accounts for the continuity of the velocity potential upstream of the blades, two new terms $D^{(3)}$ and $D^{(4)}$ are added and coupled to get the full solution by an infinite matrix system. In a first step, $D^{(1)}$ is computed, then $D^{(2)}$ and finally, in the third step, $D^{(3)}$ and $D^{(4)}$ in a coupled fashion. The technique has been described previously by Glegg (1999) for a vortical gust interacting with a blade row. The expressions of $\zeta$ and of the inter-blade phase angle $\sigma$ are given in Appendix A.1. By linearity of the problem, the scattered field will be harmonic in time at the same frequency $\omega_{e x}$ and since the blades have infinite span, it will also be harmonic in the spanwise direction $\boldsymbol{u}_{z_{c}}$ and can be defined as

$$
\widehat{\phi}\left(x_{c}, y_{c}, z_{c}, t\right)=\phi\left(x_{c}, y_{c}\right) \exp \left(-\mathrm{i} \omega_{e x} t+\mathrm{i} k_{z_{c 0}} z_{c}\right) .
$$

Glegg's formulation provides the acoustic field outside the cascade with no need for explicitly calculating the unsteady loadings on the blades and the field between adjacent blades. This is all the more important as the pressure jump evaluation is often time-consuming and a source of errors. Boquilion et al. (2003) and Grissom, Devenport \& Glegg (2005) investigated the distortion of a velocity field passing through a blade row and the velocity potential field inside the inter-blade channels. Their predictions were compared to measurements made in the cases of a homogeneous incident turbulence and of a discrete upwash gust, respectively. These comparisons were performed by numerically computing the integral of (2.2), whereas the radiated field outside the blade row was provided by the Wiener-Hopf procedure. It would be more attractive to also produce the velocity potential of the scattered field inside the inter-blade channel analytically, to make the understanding of the cascade mechanisms easier.

Besides, the pressure jump along the blade chord may be needed as the explicit equivalent noise-source distribution if the radiated field is to be determined by application of the acoustic analogy (Ffowcs-Williams \& Hawkings 1969). Though cumbersome at a first glance, such a procedure is necessary when resorting to a rectilinear cascade response function to deal with the three-dimensional geometry of a real fan stage. A strip theory is then applied to split the three-dimensional configuration into a series of equivalent rectilinear-cascade problems. The unsteady blade loading is first calculated on each strip and the modal coefficients inside the fan duct can then be determined from the contributions of all strips (Posson 2008; Posson et al. 2010).

Before going further in the formulation, different types of modes, found in the model, must be highlighted. A vortical gust impinging on the rectilinear cascade produces an acoustic field outside the cascade, which can be decomposed into modes of index $q$, called cascade modes. These modes are oblique waves in the rectilinearcascade reference frame and are related to the wavenumbers $\lambda_{q}^{ \pm}$given in (A 1). Below a non-zero frequency, here called the cascade cut-on frequency $\omega_{e x, c}$, the radiated field decays exponentially. This frequency corresponds to the smallest cut-on frequency of all possible modes. The cut-on frequency of mode $q$ is imposed by the relation 
$\kappa_{e}^{2}-f_{q}^{2}=0$. If the cascade is an unwrapped model of a blade row in an annular duct, the radiated mode $q$ is related to the duct modes of azimuthal order $m \times m=m_{g}-q B$, where the incident inter-blade phase angle is $\sigma=2 \pi m_{g} / B$ and $B$ is the number of blades. The position of the incident gust frequency with respect to the successive cascade radiated modes will modify both the radiated field and the unsteady blade loading, in particular close to the cascade cut-on frequency. Furthermore, the pressure field between adjacent blades is a combination of the characteristic modes of the interblade channel. These modes are related to the wavenumbers $\delta_{n}$ and $\varepsilon_{l}$ in the model, given by (A 1).

The subsequent analytical developments are considered only for the reference channel $y_{c} \in\left[0, h\right.$ [ and the results for other values of $y_{c}$ can be deduced from the periodicity of the cascade and the inter-blade phase angle $\sigma$. Let $A_{c}$ denote half the term in the curly brackets of (2.2):

$$
A_{c}\left(k_{x_{c}}, y_{c}\right)=+\frac{1}{2}\left\{\frac{\mathrm{e}^{\mathrm{i} \zeta y_{c}}}{1-\exp \left(\mathrm{i} \zeta h-\mathrm{i} k_{x_{c}} d-\mathrm{i} \sigma\right)}+\frac{\mathrm{e}^{-\mathrm{i} \zeta y_{c}}}{1-\exp \left(-\mathrm{i} \zeta h-\mathrm{i} k_{x_{c}} d-\mathrm{i} \sigma\right)}\right\} .
$$

The integral in (2.2) can be performed by applying the residue theorem, paying special attention to all terms, because the different contributions $D^{(1)}-D^{(4)}$ of $D$ and of the factor $A_{c}$ do not behave similarly for field points everywhere inside the inter-blade channel. This means that a unique contour of integration in the complex plane cannot be uniformly valid for the whole product $D \times A_{c}$. In order to determine the velocity potential of the scattered field, $D$ and $A_{c}$ are written in a more convenient way. $D$ is first split into two parts: $D^{(1)}+D^{(3)}$ and $D^{(2)}+D^{(4)}$. As pointed out by Glegg (1999), the acoustic field upstream of the blade row is related to $D^{(1)}+D^{(3)}$ only and the downstream field involves only $D^{(2)}+D^{(4)}$. However, all terms contribute inside the inter-blade channel. It is also convenient to rewrite the term $A_{c}$ found in (2.4) in the equivalent form

$$
A_{c}\left(k_{x_{c}}, y_{c}\right)=A_{c}^{(1)}\left(k_{x_{c}}, y_{c}\right)+A_{c}^{(2)}\left(k_{x_{c}}, y_{c}\right),
$$

with

and

$$
A_{c}^{(1)}\left(k_{x_{c}}, y_{c}\right)=-\frac{\exp \left(\mathrm{i}\left(k_{x_{c}} d+\sigma\right)\right) \cos \left(\zeta y_{c}\right)}{2\left(\cos (\zeta h)-\cos \left(k_{x_{c}} d+\sigma\right)\right)}
$$

$$
A_{c}^{(2)}\left(k_{x_{c}}, y_{c}\right)=\frac{\cos \left(\zeta\left(y_{c}-h\right)\right)}{2\left(\cos (\zeta h)-\cos \left(k_{x_{c}} d+\sigma\right)\right)} .
$$

Equation (2.5) will be used as such or factorized according to the Wiener-Hopf splitting functions $J_{+}$and $J_{-}$alternatively (noting that $1 / J_{+}=J_{-} / j$, where $j$ and $J_{ \pm}$ are defined in (A 4) and (A 3)), depending on the chordwise position in the inter-blade area and whether the contribution $D^{(1)}+D^{(3)}$ or $D^{(2)}+D^{(4)}$ is calculated. For this procedure, the inter-blade area for staggered blades is divided into three rectangular patches, as proposed by Peake (1993) for the response function to a two-dimensional incident gust. The three patches for the strip $y_{c} \in[0, h[$ are defined by

$$
\left.\begin{array}{rr}
0<x_{c}<d, & \text { patch (a), } \\
d \leqslant x_{c} \leqslant c, & \text { patch (b), } \\
c<x_{c}<c+d, & \text { patch (c), }
\end{array}\right\}
$$

and are shown in figure 2. The middle overlap area (b) necessary to couple the leading-edge and trailing-edge diffractions in the model is treated as a short duct, 
(a) Case A: $d<c / 2$

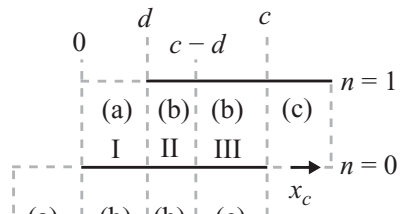

(a) (b) (b) (c) (b) Case B: $d>c / 2$

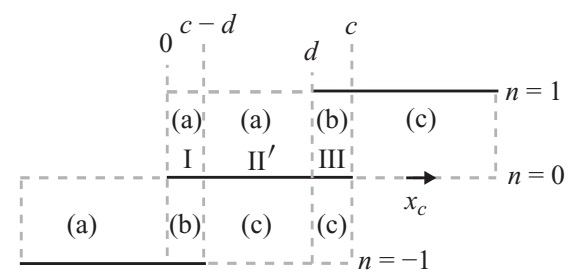

FIGURE 2. Subdivision of the inter-blade space into patches $(\mathrm{a}, \mathrm{b}, \mathrm{c})$ and into segments: (I, II, III) in the case A where $0<d<c / 2$; (I, II', III) in the case B where $c / 2<d<c$. Patches and segments are defined for the analytical calculation of the acoustic field and of the pressure jump on the reference blade, respectively.

whereas the other two have an intermediate behaviour between free space and a waveguide, with interactions between the cascade radiated modes associated with $\lambda_{q}^{ \pm}$, and the inter-blade channel duct modes associated with $\delta_{n}$ and $\varepsilon_{l}$. The mathematical statement imposes rectangular patches (a) and (c) instead of the triangles that could be defined intuitively from the leading-edge front of the cascade.

The question of the closure of the integration path must be re-addressed for each patch to ensure the convergence of the integral. As an example, the solution is detailed here for patch (a) for the leading-edge term $\Phi^{+}\left(k_{x_{c}}, y_{c}\right)=\left(D^{(1)}\left(k_{x_{c}}\right)+\right.$ $\left.D^{(2)}\left(k_{x_{c}}\right)\right) \times A_{c}\left(k_{x_{c}}, y_{c}\right)$. The velocity potential of the scattered field will be decomposed into two parts. One is $\phi_{a c}$, the acoustic part of $\phi$. The other one, $\phi_{v}$, is the pressure-free part of the potential convected by the mean flow, which corresponds to the vortical disturbance produced to ensure the rigid-wall boundary condition on blades and to the vortical gust produced to fulfil the Kutta condition at the blades' trailing edge. The two terms $A_{c}^{(1)}$ and $A_{c}^{(2)}$ have different behaviours as $\left|k_{x_{c}}\right|$ tends to infinity. The variable $\Phi^{+}\left(k_{x_{c}}, y_{c}\right)$ can be written as one of the following two expressions (see (2.7) and (2.8)) using (2.5) and the definition of $J^{+}$and $j$ :

$$
\begin{aligned}
\Phi^{+}\left(k_{x_{c}}, y_{c}\right) & =\left(D^{(1)}\left(k_{x_{c}}\right)+D^{(3)}\left(k_{x_{c}}\right)\right) A_{c}\left(k_{x_{c}}, y_{c}\right) \\
& =\Phi_{1}^{+}\left(k_{x_{c}}, y_{c}\right)+\Phi_{2}^{+}\left(k_{x_{c}}, y_{c}\right),
\end{aligned}
$$

with

$$
\Phi_{1}^{+}\left(k_{x_{c}}, y_{c}\right)=\left(D^{(1)}\left(k_{x_{c}}\right)+D^{(3)}\left(k_{x_{c}}\right)\right) A_{c}^{(1)}\left(k_{x_{c}}, y_{c}\right),
$$

and

$$
\Phi_{2}^{+}\left(k_{x_{c}}, y_{c}\right)=\left(D^{(1)}\left(k_{x_{c}}\right)+D^{(3)}\left(k_{x_{c}}\right)\right) A_{c}^{(2)}\left(k_{x_{c}}, y_{c}\right)
$$

or

$$
\Phi^{+}\left(k_{x_{c}}, y_{c}\right)=\Phi_{1}^{+}\left(k_{x_{c}}, y_{c}\right)+\Phi_{2}^{+}\left(k_{x_{c}}, y_{c}\right),
$$

with

$$
\begin{aligned}
\Phi_{1}^{+}\left(k_{x_{c}}, y_{c}\right)=\left\{\frac{\mathrm{i} w_{0}}{(2 \pi)^{2} J_{-}\left(-k_{x_{c} 0}\right)\left(k_{x_{c}}+k_{x_{c} 0}\right)}+\sum_{l \geqslant 1} \frac{B_{l} J_{+}\left(\varepsilon_{l}\right)}{k_{x_{c}}-\varepsilon_{l}}\right\} \\
\times J_{-}\left(k_{x_{c}}\right) \frac{2 \pi \exp \left(\mathrm{i}\left(\sigma+k_{x_{c}} d\right)\right) \cos \left(\zeta y_{c}\right)}{\zeta \sin (\zeta h)},
\end{aligned}
$$




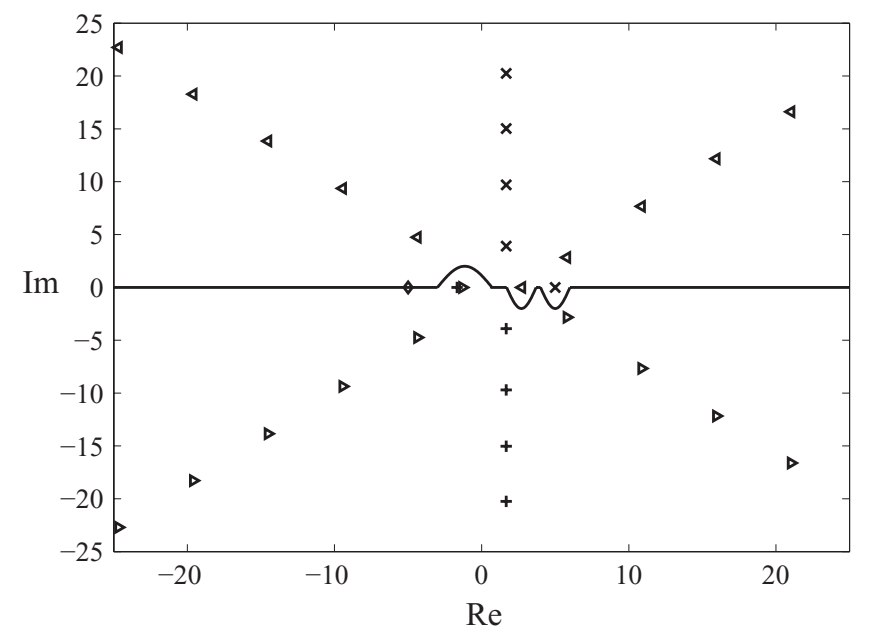

Figure 3. Position of the poles and zeros exhibited in the model: $\left(\lambda_{q}^{+}\right)_{q \in \mathbb{Z}}(\triangleleft),\left(\lambda_{q}^{-}\right)_{q \in \mathbb{Z}}(\triangleright)$, $\left(\varepsilon_{l}\right)_{l \geqslant 1}(\mathbf{\times}),\left(\delta_{n}\right)_{\geqslant 1}(+)$ and $-k_{x_{c 0}}(\diamond)$ for the test case defined in table 1. Sketch of a part of the contour of integration in the complex plane by-passing poles on the real axis $(-)$.

and

$$
\begin{aligned}
& \Phi_{2}^{+}\left(k_{x_{c}}, y_{c}\right)=\left\{\frac{-\mathrm{i} w_{0}}{(2 \pi)^{2} J_{-}\left(-k_{x_{c 0}}\right)\left(k_{x_{c}}+k_{x_{c} 0}\right)}\right.\left.-\sum_{l \geqslant 1} \frac{B_{l} J_{+}\left(\varepsilon_{l}\right)}{k_{x_{c}}-\varepsilon_{l}}\right\} \\
& \times J_{-}\left(k_{x_{c}}\right) \frac{2 \pi \cos \left(\zeta\left(y_{c}-h\right)\right)}{\zeta \sin (\zeta h)} .
\end{aligned}
$$

As the splitting functions $J_{ \pm}$have an algebraic growth, $\Phi_{1}^{+}\left(k_{x_{c}}, y_{c}\right) \exp \left(-\mathrm{i} k_{x_{c}} x_{c}\right)$ behaves like $\exp \left(\mathrm{i}_{x_{c}}\left(d-x_{c}\right)\right)$ at infinity, whereas $\Phi_{2}^{+}\left(k_{x_{c}}, y_{c}\right) \exp \left(-\mathrm{i} k_{x_{c}} x_{c}\right)$ behaves like $\exp \left(-\mathrm{i} k_{x_{c}} x_{c}\right)$. Since $0<x_{c}<d$ in patch (a), the contours for $\Phi_{1}^{+}\left(k_{x_{c}}, y_{c}\right) \exp \left(-\mathrm{i} k_{x_{c}} x_{c}\right)$ and $\Phi_{2}^{+}\left(k_{x_{c}}, y_{c}\right) \exp \left(-\mathrm{i} k_{x_{c}} x_{c}\right)$ must be closed in the upper and lower parts of the complex plane, respectively, as shown in figure 3. As $J_{-}$is analytic in the lower half-plane only, the expression $(2.7 b)$ is chosen to compute the inverse Fourier transform of $\Phi_{1}^{+}\left(k_{x_{c}}, y_{c}\right)$ and the expression (2.8c) to compute the inverse Fourier transform of $\Phi_{2}^{+}\left(k_{x_{c}}, y_{c}\right)$. Then

$$
\Phi_{1}^{+}\left(k_{x_{c}}, y_{c}\right)=-\left[D^{(1)}\left(k_{x_{c}}\right)+D^{(3)}\left(k_{x_{c}}\right)\right] \underbrace{\frac{\exp \left(\mathrm{i}\left(k_{x_{c}} d+\sigma\right)\right) \cos \left(\zeta y_{c}\right)}{2\left(\cos (\zeta h)-\cos \left(k_{x_{c}} d+\sigma\right)\right)}}_{\left(1^{\prime} a c\right)} .
$$

Inside the contour, $D^{(3)}$ has simple poles at $\left(\varepsilon_{l}\right)_{l \in \mathbb{N}^{*}}$ and the factor $\left(1^{\prime} a c\right)$ of (2.9) has poles at the zeros $\lambda_{q}^{-}$of the function $k_{x_{c}} \mapsto \cos (\zeta h)-\cos \left(k_{x_{c}} d+\sigma\right)$ in the upper part of the complex plane. The residue theorem finally yields the inverse Fourier transform as

$$
\phi_{a c, a, 1}^{+}\left(x_{c}, y_{c}\right)=\sum_{q \in \mathbb{Z}} \phi_{a c, a, 1, \lambda, q}^{+}\left(x_{c}, y_{c}\right)+\sum_{l \geqslant 1} \phi_{a c, a, 1, l}^{+}\left(x_{c}, y_{c}\right),
$$

with

$$
\phi_{a c, a, 1, \lambda, q}^{+}\left(x_{c}, y_{c}\right)=-\mathrm{i} \pi \zeta_{q}^{+} D\left(\lambda_{q}^{+}\right) \frac{\cos \left(\zeta_{q}^{+} y_{c}\right) \exp \left(\mathrm{i}\left(\sigma+\lambda_{q}^{+} d\right)\right) \exp \left(-\mathrm{i} \lambda_{q}^{+} x_{c}\right)}{\sin \left(\lambda_{q}^{+} d+\sigma\right)\left(\zeta_{q}^{+} d+\mathscr{S}_{q}^{+} h \beta^{2} \eta_{q}^{+}\right)},
$$


and

$$
\phi_{a c, a, 1, l}^{+}\left(x_{c}, y_{c}\right)=\mathrm{i} \pi \frac{B_{l} \exp \left(\mathrm{i}\left(\varepsilon_{l}\left(d-x_{c}\right)+\sigma\right)\right) \cos \left(\zeta\left(\varepsilon_{l}\right) y_{c}\right)}{\left(\cos \left(\zeta\left(\varepsilon_{l}\right) h\right)-\cos \left(\varepsilon_{l} d+\sigma\right)\right)} .
$$

The duct modes $\left(\varepsilon_{l}\right)_{l} \geqslant 1$ propagating upstream appear here because step 3 of the problem introduces the backscattering by the leading edges (terms in $B_{l}$ ) acting on the field scattered from the trailing edge (terms in $A_{n}$ ).

Concerning the term $\Phi_{2}^{+}\left(k_{x_{c}}, y_{c}\right)$, the factor in the curly brackets of $(2.8 c)$ has a simple pole at $-k_{x_{c 0}}=-\omega_{g} / U_{c}$, in the case of an incident vortical gust, leading to the contribution $\phi_{v, a, 2,1}$ :

$$
\phi_{v, a, 2,1}^{+}\left(x_{c}, y_{c}\right)=-\frac{w_{0} \cos \left(\zeta\left(-k_{x_{c 0}}\right)\left(y_{c}-h\right)\right) \mathrm{e}^{\mathrm{i} k_{x_{c 0}} x_{c}}}{\zeta\left(-k_{x_{c 0}}\right) \sin \left(\zeta\left(-k_{x_{c} 0}\right) h\right)} .
$$

Other simple poles are found at the zeros $\left(\delta_{n}\right)_{n \in \mathbb{N}^{*}}$ of the function $k_{x_{c}} \mapsto(\zeta \sin (\zeta h))^{-1}$ in the lower half-plane. The contribution to the acoustic potential then reads

$$
\phi_{a c, a, 2}^{+}\left(x_{c}, y_{c}\right)=\sum_{n \geqslant 1} \phi_{a c, a, 2, n}^{+}\left(x_{c}, y_{c}\right)+\sum_{n \geqslant 1} \sum_{l \geqslant 1} \phi_{a c, a, 2, n, l}^{+}\left(x_{c}, y_{c}\right),
$$

with

and

$$
\phi_{a c, a, 2, n}^{+}\left(x_{c}, y_{c}\right)=\frac{-w_{0} J_{-}\left(\delta_{n}\right) \cos \left(\zeta\left(\delta_{n}\right) y_{c}\right) \mathrm{e}^{-\mathrm{i} \delta_{n} x_{c}}}{J_{-}\left(-k_{x_{c} 0}\right)\left(\delta_{n}+k_{x_{c} 0}\right) \beta^{2} h d_{n} \vartheta_{n-1}},
$$

$$
\phi_{a c, a, 2, n, l}^{+}\left(x_{c}, y_{c}\right)=\mathrm{i}(2 \pi)^{2} \frac{B_{l} J_{+}\left(\varepsilon_{l}\right) J_{-}\left(\delta_{n}\right) \cos \left(\zeta\left(\delta_{n}\right) y_{c}\right) \mathrm{e}^{-\mathrm{i} \delta_{n} x_{c}}}{\left(\delta_{n}-\varepsilon_{l}\right) \beta^{2} h d_{n} \vartheta_{n-1}} .
$$

Finally, the acoustic field associated with the leading-edge terms $D^{(1)}+D^{(3)}$ is the sum of $(2.10 a)$ and $(2.12 a)$. The contribution of the trailing-edge terms $D^{(2)}+D^{(4)}$ and the formulation in patches (b) and (c) can be derived in a similar way (Posson 2008). The expressions valid on the three patches and the different terms of $D$ and $A_{c}$ are listed in (A 17) and (A 18) of Appendix A.2 for the acoustic part of the scattered potential field.

The acoustic field in the inter-blade area carries information on the blade coupling inside the cascade which is hard to analyse with lifting-surface methods. Nevertheless, the ultimate aim of the present model is to compute the broadband noise of a three-dimensional annular blade row. Rather than the acoustic field in the inter-blade channels, the main issue is the pressure jump on the blades to be used as a source term in the acoustic analogy for in-duct propagation. This pressure jump is often calculated by collocation methods and as such is very sensitive to the collocation accuracy and time-consuming. This is why the aforementioned analytical extension is now used to provide closed-form expressions for the potential jump $\Delta \phi$ and then the unsteady loading $\Delta p_{0}\left(x_{c}\right)=p\left(x_{c}, 0^{+}\right)-p\left(x_{c}, 0^{-}\right)$is obtained according to

$$
\Delta p_{0}\left(x_{c}\right) \exp \left(-\mathrm{i} \omega_{e x} t+\mathrm{i} k_{z_{c}} z_{c}\right)=-\rho_{0} \frac{\mathrm{D}}{\mathrm{D} t}\left(\Delta \phi\left(x_{c}\right) \exp \left(-\mathrm{i} \omega_{e x} t+\mathrm{i} k_{z_{c}} z_{c}\right)\right) .
$$

The potential jump for the reference blade at $y_{c}=0$ reads

$$
\Delta \phi\left(x_{c}\right)=\phi_{0}\left(x_{c}, 0^{+}\right)-\phi_{-1}\left(x_{c}, 0^{-}\right),
$$

where the index $n$ of $\phi_{n}$ denotes the channel for which $y_{c} \in[n h,(n+1) h]$. Since the potential in channel $n$ is related to the one in the reference channel $\phi_{0}$ by

$$
\phi_{n}\left(x_{c}, y_{c}\right)=\phi_{0}\left(x_{c}-n d, y_{c}-n h\right) \mathrm{e}^{\mathrm{i} n \sigma},
$$


the potential jump is defined by the equation

$$
\Delta \phi\left(x_{c}\right)=\phi_{0}\left(x_{c}, 0^{+}\right)-\phi_{0}\left(x_{c}+d, h^{-}\right) \mathrm{e}^{-\mathrm{i} \sigma} .
$$

Once the potential field in the reference channel is defined on the three patches ( $\mathrm{a}$, $\mathrm{b}$ and $\mathrm{c}$ ) according to formulae (A 14)-(A 18) of Appendix A.2, the pressure jump is derived for the three segments, I, II, III or I, II', III. The result depends on the positions of the points $0^{+}$and $0^{-}$in the channels and on the nature of patches on both sides of the blade ( $\mathrm{a}, \mathrm{b}$ or $\mathrm{c}$ ). In other words, it depends on the sign of $c / 2-d$, as depicted in figures $2(a)$ and $2(b)$, where the intermediate segments (II) and (II') are surrounded by patches of types (b) and (b), and types (a) and (c), respectively. The final expressions for the pressure jump are also listed in Appendix A.3.

The whole problem must be revisited in the particular case where blades are not staggered $(d=0)$ and the incident gust impinges on all blades in phase $(\sigma \equiv 0[2 \pi])$. The former solution is no longer valid and would diverge if used directly due to removable singularities. A new formulation can be given in the case where the poles and zeros have changed. Formulae for this case are given in Appendix B for the sake of completeness.

Results are presented below for test cases with zero sweep and lean angles $\left(\varphi=0^{\circ}\right.$; $\psi=0^{\circ}$.

\section{Unsteady blade loading}

The aforementioned model of cascade response function is applied to the problem of the sound generated by a vortical gust impinging on a rectilinear cascade. The unsteady loading on the blades is investigated first. The validity of the proposed extension is checked by comparing the results with alternative semi-numerical rectilinear-cascade models and also with three-dimensional linearized Euler codes available in the literature. A comparison is also made with the unsteady blade loading of an isolated flat plate to assess the cascade effect. The quantities of interest will be made non-dimensional with respect to the blade chord $c$, the flow density $\rho_{0}$ and the sound speed $c_{0}$ and noted with overbars. Particular non-dimensional factors will be specifically defined when needed.

\subsection{Pressure jump across the blades}

The analytical solution is first compared to the modified version of Whitehead's LINSUB computer code (e.g. Whitehead 1987), based on the semi-numerical model developed by Smith (1973). This model was used by Hanson $(1994,1999)$ and is currently applied in broadband-noise-prediction schemes such as those developed by Cheong, Joseph \& Soogab (2006) or Lloyd \& Peake (2008). The response of the blade row to any excitation (bending or torsional vibration, acoustic or vortical incident gust) is given by bounded and free vortices distributed along the blades and their wakes. The comparison is performed on a staggered benchmark configuration, defined in table 1 and presented by Verdon \& Hall (1990) to validate the linearized Euler code linearized inviscid flow (LINFLO) (Hall \& Verdon 1991) against LINSUB in a flat-plate configuration. The results for the real and imaginary parts of the pressure jump made non-dimensional by the factor $\rho_{0} w_{0} U_{0}$ are plotted in figures $4(a)$ and $4(b)$. A very good agreement is found between the analytical and LINSUB results for all tested Mach numbers.

The present model based on Glegg's formulation also enables computing the unsteady blade loading produced by the interaction of the rectilinear cascade with 


$\begin{array}{cc}\bar{s} & 1 \\ \chi(\text { deg. }) & 45 \\ M & \{0.3 ; 0.5 ; 0.7\} \\ \bar{\omega}_{e x} & 5 M \\ \sigma & -2 \pi \\ \bar{k}_{z_{c 0}} & 0 \\ \varphi & 0\end{array}$

TABLE 1. Input parameters of the test case defined by Verdon \& Hall (1990), used in figure 4.
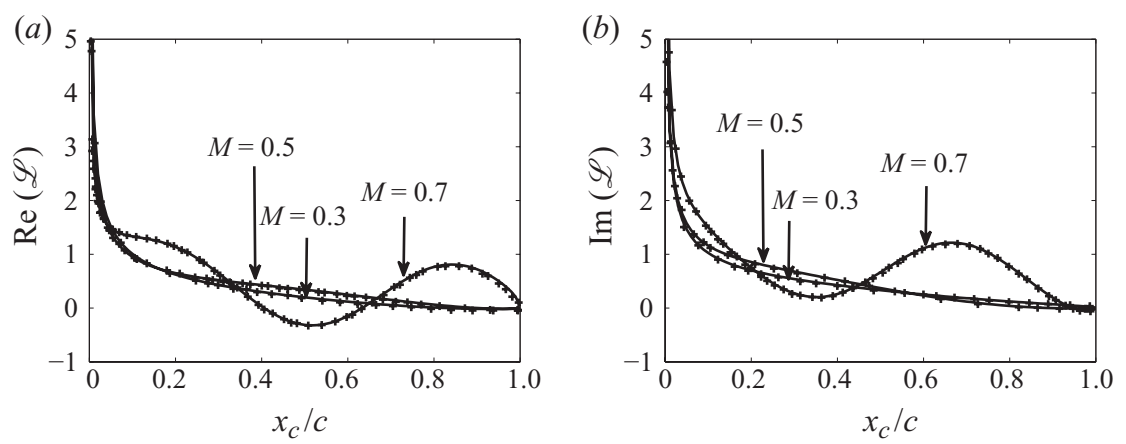

FIGURE 4. Chordwise distributions of the unsteady blade loading $-\Delta p_{0}$ made non-dimensional by the factor $\rho_{0} w_{0} U_{0}: \mathscr{L}=-\Delta p_{0} /\left(\rho_{0} w_{0} U_{0}\right)$ from the analytical formulation of $\S 2(-)$ and LINSUB code (+) at $M=0.3, M=0.5$ and $M=0.7$. (a) Real part; (b) imaginary part. Test case of table 1. Results are the complex conjugate of those presented by Verdon \& Hall (1990) because a different convention for the time Fourier transform is used.

a three-dimensional gust. Goldstein (1976, chapter 5) developed a rectilinear-cascade model for the case that accounts for the presence of parallel duct walls. Comparisons can then be performed with the semi-numerical LINC code later developed by Atassi \& Hamad (1981), first with a two-dimensional gust and second with a threedimensional one. The benchmark test for the two-dimensional gust corresponds to a narrow-annulus configuration presented by Elhadidi \& Atassi (2002). This case can either be investigated with the three-dimensional linearized Euler code (e.g. Atassi et al. 2004) based on the real geometry, or with semi-numerical models (e.g. Atassi \& Hamad 1981) and analytical rectilinear models at the mean radius $R_{m}=0.99$. The input parameters are reported in table 2 where the frequency is made non-dimensional with respect to the mean radius $R_{m}: \widetilde{\omega}_{e x}=\omega_{e x} R_{m} / c_{0}=\bar{\omega}_{e x} B_{S} \bar{s} /(2 \pi)$. The unsteady vane loading is now made non-dimensional by dividing by the factor $\rho_{0} c_{0}^{2}$. A phase shift equal to $-k_{x_{c 0}} c / 2$ is introduced to be consistent with the benchmark test in which the chordwise origin is defined at mid-chord rather than at the leading edge. The predictions obtained in the mid-frequency range for $\widetilde{\omega}_{e x}=6.5 \pi$ by Posson $\&$ Roger (2007) show that the two rectilinear codes are in a very close agreement and suggested the present comparisons involving three-dimensional gusts. Besides, the agreement between the codes is good not only in the mid-frequency range $\left(\widetilde{\omega}_{e x}=6.5 \pi\right)$ where two acoustic cascade modes are cut-on, but also at the low frequency, $\widetilde{\omega}_{e x}=0.5 \pi$, below the cascade cut-on frequency, as shown in figure $5(a)$. Both results also agree well with the computations by Elhadidi \& Atassi (2002) based on the numerical code developed by Atassi et al. (2004). The small remaining discrepancies very close to the leading edge are most likely attributed to resolution issues in the Euler 


$\begin{array}{cc}\bar{s} & 1 \\ \chi(\mathrm{deg} .) & 45 \\ M & 0.509 \\ \widetilde{\omega}_{e x}=B_{S} \bar{s}_{e x} / 2 \pi & 0.5 \pi \\ \sigma & 2 \pi B_{R} / B_{S} \\ \bar{k}_{z_{c 0}} & 0 \\ \varphi & 0 \\ B_{S} & 24 \\ B_{R} & 16 \\ R_{T} & 1 \\ R_{H} / R_{T} & 0.98\end{array}$

TABLE 2. Input parameters of the case defined by Elhadidi \& Atassi (2002), used in figure 5.
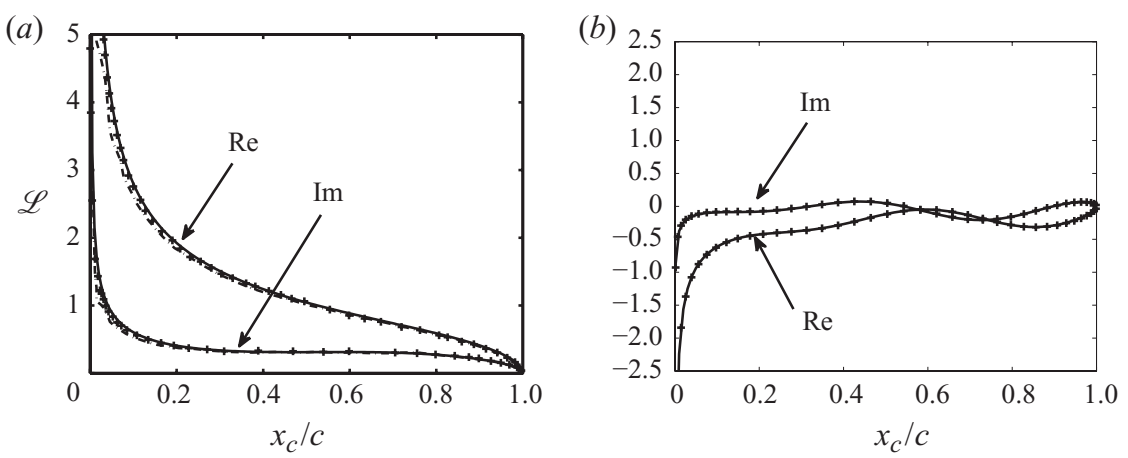

Figure 5. Chordwise distributions of the real and imaginary parts of the unsteady vane loading $-\Delta p_{0}$ for an incident upwash amplitude at mid-span and mid-chord $a_{0}=c_{0}$ $\left(w_{0}=a_{0} \exp \left(-\mathrm{i} k_{x_{c 0}} c / 2\right)\right)$. (a) The unsteady blade loading is made non-dimensional by the factor $\rho_{0} c_{0}^{2}: \mathscr{L}=-\Delta p_{0} \exp \left(-\mathrm{i} k_{x_{c} 0} c / 2\right) /\left(\rho_{0} c_{0}^{2}\right)$ from the analytical formulation of $\S 2(-)$, the numerical three-dimensional linearized Euler code of Atassi et al. (2004) (-. - .) and the LINC code $(+) ; \widetilde{\omega}_{e x}=0.5 \pi$ (test case of table 2 ). (b) The unsteady blade loading is made non-dimensional by the factor $\rho_{0} a_{0} U_{0}: \mathscr{L}=-\Delta p_{0} \exp \left(-\mathrm{i} k_{x_{c 0}} c / 2\right) /\left(\rho_{0} a_{0} U_{0}\right)$ from the analytical formulation of $\S 2(-)$ and the LINC code $(+)$ for the case defined in table 3.

numerical method. They are small enough to have negligible effects on a broadband noise prediction. The good agreement is a first indication of the reliability of the rectilinear-cascade model in a narrow-annulus configuration.

The present expression is finally compared with an updated version of the LINC code for a three-dimensional gust. The proposed test case is taken from a set of comparisons performed with M. M. Logue \& H. M. Atassi (personal communication on rectilinear-cascade models, 2006). The input parameters are given in table 3 and the two predictions of the unsteady blade loading are plotted in figure 5(b). Again the very good agreement shows that the three-dimensionality of the incident gust is properly included in the analytical solution.

\subsection{Comparison with an isolated-airfoil response function}

Analytical models based on an isolated-airfoil response function only take into account a part of the cascade effects by the imposed inter-blade phase angle, whereas the distributed unsteady lift is derived by ignoring adjacent blades. The first developments on unsteady-airfoil theory were derived for incompressible flows by Sears (1941). He derived an analytical expression for the unsteady lift of an 


$\begin{array}{cc}\bar{s} & 1 \\ \chi(\text { deg. }) & 30 \\ M & 0.5 \\ \varphi & 0 \\ k_{1} & 10 \\ k_{2} & 2 \\ k_{3} & 5 \\ \bar{\omega}_{e x} & 2 k_{1} M=10 \\ \bar{k}_{y_{c 0}} & 2 k_{2}=4 \\ \bar{k}_{z_{c} 0} & 2 k_{3}=10\end{array}$

TABLE 3. Input parameters of the test case defined with M. M. Logue \& H. M. Atassi (personal communication on rectilinear-cascade models, 2006) to compare the two rectilinear models for a three-dimensional gust with the definition of the gust wavenumbers $\left(k_{1}, k_{2}, k_{3}\right)$ in the LINC code, used in figure $5(b)$.

airfoil in incompressible non-uniform flows, from the theory of von Kármán \& Sears (1938). After the earlier analyses of the compressible subsonic problem by Possio (1938) and Reissner (1951) notably, Graham (1970) constructed similarity rules for the load distributions of a thin airfoil to relate a general case to the twodimensional compressible case and the three-dimensional incompressible case. Among the developed closed-form approximate analytical solutions, Amiet (1976) proposed a high-frequency asymptotic solution. In order to quantify the expected errors in such isolated-airfoil approaches, especially when the blades are widely spaced and the overlap tends to zero, the present cascade solution is compared, for the same vortical disturbance, both to Sears' low-frequency incompressible solution and to Amiet's high-frequency compressible solution with trailing-edge backscattering (e.g. Amiet 1975). At high frequencies, Amiet's solution is a priori equivalent to retaining only the first iterations $D^{(1)}$ and $D^{(2)}$ of the function $D$ in the limit of vanishing solidity. In that sense, the comparison is relevant, since the corrections $D^{(3)}$ and $D^{(4)}$ are important to properly account for only those resonance effects which do not occur on an isolated airfoil. It should also be noted that the inter-blade phase angle or equivalently the wavenumber $k_{y_{c 0}}$ normal to a blade is not involved in isolatedairfoil theories. Unsteady blade loading results are plotted in figures $6(a)$ and $6(b)$ for various solidities at fixed inter-blade phase angle $\sigma=3 \pi / 4$ and stagger angle, and in figure 7 for various inter-blade phase angles and a fixed solidity $\Gamma=c / h=1.2$ and stagger angle $\chi=20^{\circ}$. In most configurations, the cascade effect on the unsteady blade loading is significant, as depicted in figures $6(a)$ and $6(b)$. Particular resonant configurations exhibit much higher unsteady lift amplitudes, typically for $\Gamma=1.25$ in figure $6(b)$. The inter-blade phase angle can also notably modify the response, as shown in figure 7. However, as the blade overlap goes to zero with a small or moderate solidity, the unsteady blade loading gets closer to the response of an isolated airfoil (figures $6 a$ and $6 b$ for $\Gamma=1.22$ and $\Gamma=0.37$, respectively). In addition, for small overlap cases, the similarity with the isolated airfoil is often better for $\sigma=\pi / 2$, as shown in figure $7(b)$.

The effect of the Mach number on the cascade response function is studied next. First, a comparison is made at a very low Mach number, $M=0.01$, with Amiet's response and with Sears' incompressible response function in figure 8. At vanishing Mach number, the cascade response reproduces Sears' function above $k_{1}=\omega c /(2 U)=2$. Below this value, the reduced frequency $\bar{\omega}_{e x}=2 k_{1} M<0.04$ is very 

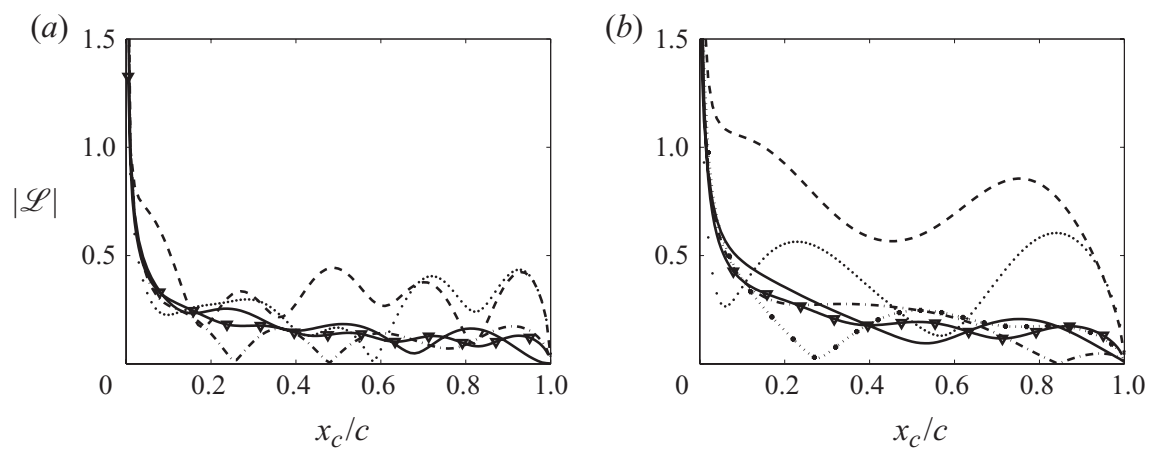

Figure 6. Chordwise amplitude distributions of the unsteady blade loading $\Delta p_{0}$ made non-dimensional by the factor $\rho_{0} w_{0} c_{0}: \mathscr{L}=\Delta p_{0} /\left(\rho_{0} w_{0} c_{0}\right)$ from the analytical formulation of $\S 2$ and from Amiet's response function $(-\nabla)$ : $(a)$ for $M=0.5, \bar{\omega}_{e x}=12, \bar{k}_{z_{c 0}}=0, \chi=50^{\circ}$, $\sigma=3 \pi / 4$, and $\Gamma=3.33(---), \Gamma=1.67(\cdots \cdots), \Gamma=1.43 \quad(-\cdot-)$ and $\Gamma=1.22(-)$;

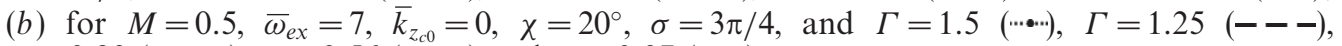
$\Gamma=0.83(\cdots \cdots), \Gamma=0.56(-\cdot-)$ and $\Gamma=0.37(-)$.
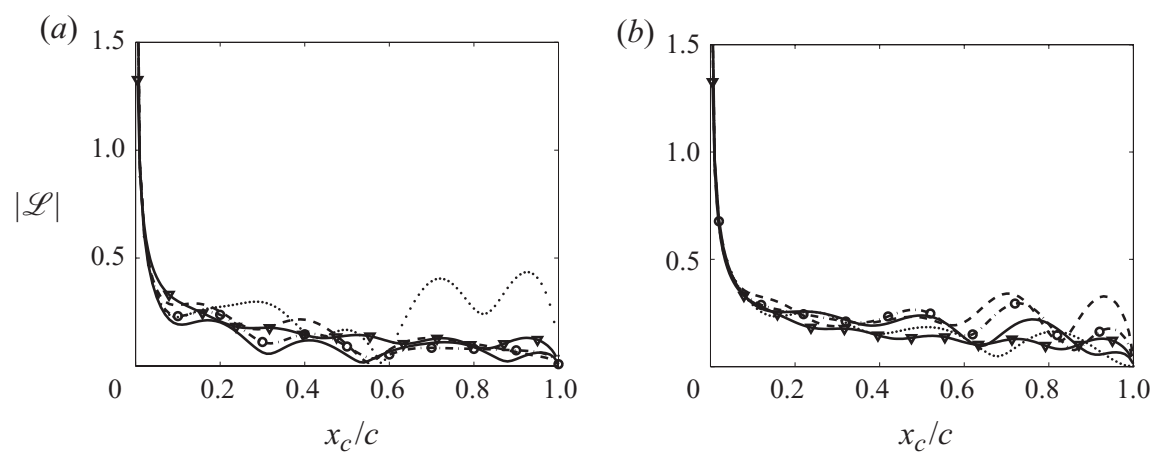

FIGURE 7. Chordwise amplitude distributions of the unsteady blade loading $\Delta p_{0}$ made non-dimensional by the factor $\rho_{0} w_{0} c_{0}: \mathscr{L}=\Delta p_{0} /\left(\rho_{0} w_{0} c_{0}\right)$ from the analytical formulation of $\S 2$ and from Amiet's response function $(-\boldsymbol{\nabla})$ for $M=0.5, \bar{\omega}_{e x}=12, \bar{k}_{z_{c 0}}=0, \chi=50^{\circ}$, with $\sigma=0$ (- - $), \sigma=\pi / 2(-), \sigma=3 \pi / 4(\cdots \cdots), \sigma=9 \pi / 4 \quad(\cdot \cdot-\circ-\cdot)$ and $(a) \Gamma=1.67$; (b) $\Gamma=1.22$.

small, and the infinite matrix system to be solved in order to compute $D$ is illconditioned. The cascade response oscillates around Sears' function. Amiet's response departs from Sears' one below much higher frequencies $\left(k_{1}=14.7\right)$. At this point, the curves superimpose but with a residual phase shift. The reduced wavenumber $k_{1}=14.7$ and Mach number $M=0.01$ lead to a parameter $\bar{\mu}=k_{1} M / \beta^{2}=0.147<\pi / 4$, which indicates, according to Amiet (1974), that the high-frequency solution should be replaced by the compressible low-frequency correction of Sears' theory.

Secondly, the blade responses are plotted in figure 9 for different reduced frequencies and three Mach numbers: $M=0.01$ (up to $M=0.1$ ) in the left column, $M=0.5$ in the centre column and $M=0.8$ in the right column. Each row in figure 9 corresponds to a particular reduced frequency: $k_{1} M=\bar{\omega}_{e x} / 2$ equal to $0.003,0.2,0.5,10$, respectively. For the very low reduced frequency $k_{1} M=0.003$, the cascade response and Sears' response are in very good agreement (figures $9 a, 9 b$ and $9 c$ ). For the quasi-incompressible case ( $M=0.01$ up to $M=0.1$ ), Amiet's response is close to these results, with a slight underestimate, even though it does not coincide until $k_{1}=14.7$, as shown previously 

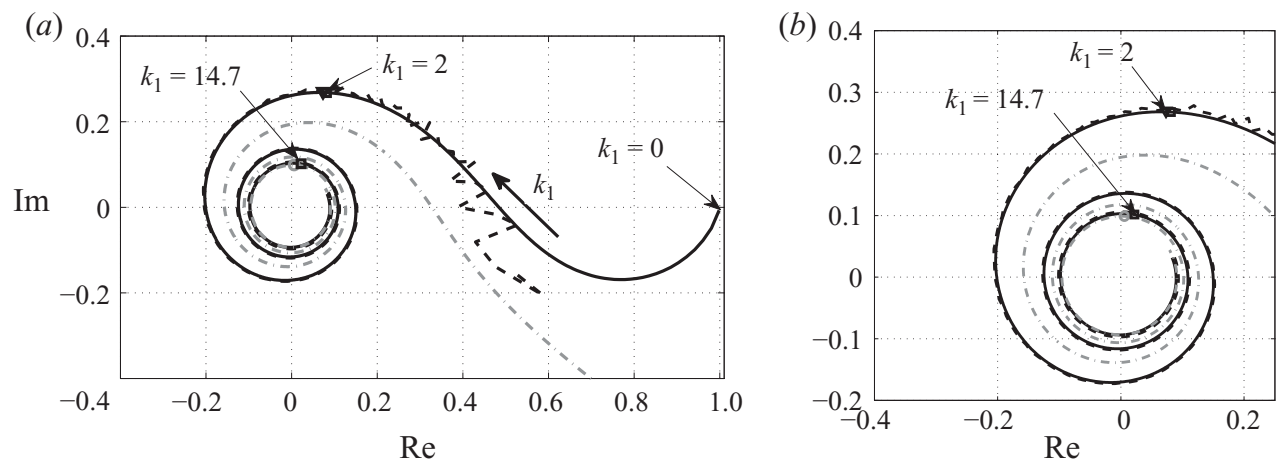

FIGURE 8. (a) Vector diagram showing the real and imaginary parts of the Sears function $(-)$ of $-\operatorname{conj}\left(C_{p}\left(k_{1}, M, \bar{d}, \chi, \sigma\right) \exp \left(-\mathrm{i} k_{1}\right)\right)$, where $C_{p}$ is the cascade unsteady lift for $M=0.01$, $\bar{d}=0.98, \chi=35^{\circ}$ and $\sigma=\pi / 2(---)$, and of Amiet's unsteady lift made non-dimensional by $\pi \rho_{0} w_{0} U_{0}$ for $M=0.01(-.-)$, versus the reduced frequency $k_{1}=\omega c /(2 U) ;(b)$ zoom around high-frequency responses.

in figure 8 and also observed in figures $9(a)$ and $9(d)$. On the contrary, for compressible cases $(M \geqslant 0.3)$, Amiet's solution highly overestimates the chordwise distribution of the lift, as shown in figures $9(b)$ and $9(c)$. As the reduced frequency is increased, Amiet's response decreases and gets closer to Sears' and cascade responses around a particular value, $k_{1} M \approx 0.2$, for almost all Mach numbers (figures $9 d, 9 e, 9 f$ ). Above this value, Sears' response still gives satisfactory results at very low Mach number (figure $9 g$ ), but no longer matches Amiet's and cascade responses in compressible cases. The discrepancy arises (figures $9 h$ and $9 i$ ) at a lower frequency as the Mach number is increased. Sears' model underestimates the response beyond $20-40 \%$ of chord with a regular drop to zero (figure $9 i$ ), whereas the leading-edge contribution leads to an overestimate of the integrated lift. When the reduced frequency $k_{1} M$ is increased, the cascade response exhibits more and more extrema along the chord (figures $9 j, 9 k, 9 l$ ), the number of which also increases with the Mach number. The maxima are reproduced by Amiet's model, but with much smaller amplitudes.

This asymptotic trend suggests that the non-zero overlap assumption inherent in the analytical model is not a serious drawback: as the overlap goes to zero, an isolatedairfoil response function can be used as a simplified solution. It is also guessed that, in the case of a small solidity, the single-airfoil theory is a good alternative. At low reduced frequencies $k_{1} M$, Sears's response can be used. At high reduced frequencies $k_{1} M$, Amiet's response is more appropriate. Even if it does not exactly capture the local extrema of the unsteady lift, the overall level is consistent and the main trend of oscillations is recovered. In view of the current plots, the relevance of the isolated response functions is less convincing at intermediate frequencies $\left(k_{1} M=0.5\right.$ in figure 9). The similarity rules of Graham (1970) could be used instead in this frequency range. The question of whether the effect on the acoustic far field is important or not will be addressed in $\S 5.2$.

\section{Acoustic field in the inter-blade channel}

The present model produces an analytical formulation for the velocity potential and then for the pressure field which are continuously valid outside and inside the inter-blade channel. To go beyond the assessment of the model, the two-dimensional 
(a) $k_{1} M=0.003, M=0.01$

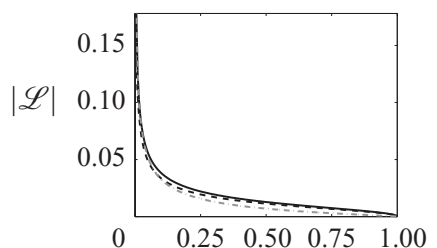

(d) $k_{1} M=0.2, M=0.01$

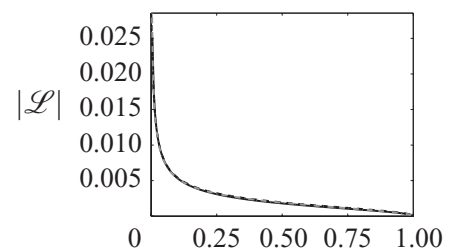

(g) $k_{1} M=0.5, M=0.01$

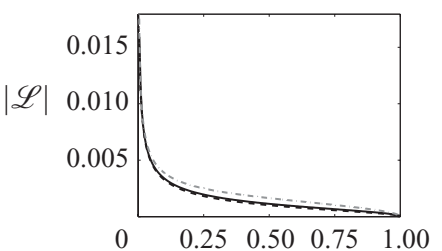

(j) $k_{1} M=10, M=0.1$

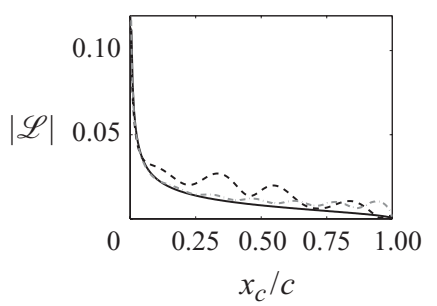

(b) $k_{1} M=0.003, M=0.5$

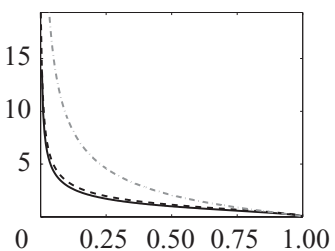

(e) $k_{1} M=0.2, M=0.5$

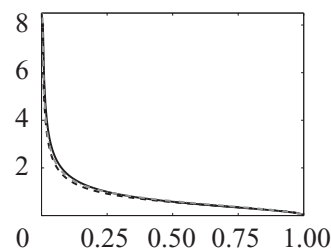

(h) $k_{1} M=0.5, M=0.5$

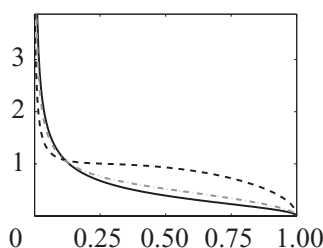

(k) $k_{1} M=10, M=0.5$

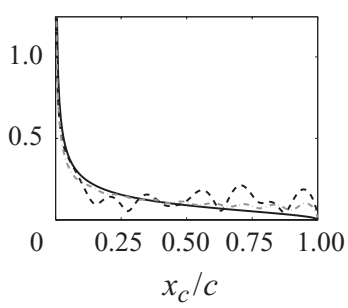

(c) $k_{1} M=0.003, M=0.8$

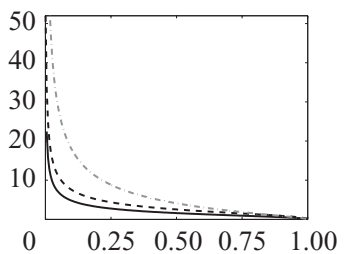

(f) $k_{1} M=0.2, M=0.8$

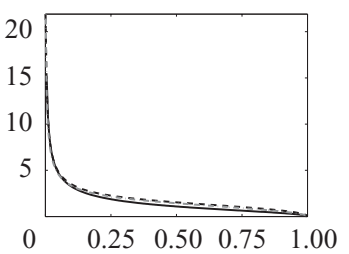

(i) $k_{1} M=0.5, M=0.8$

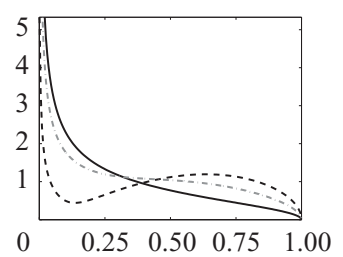

(l) $k_{1} M=10, M=0.8$

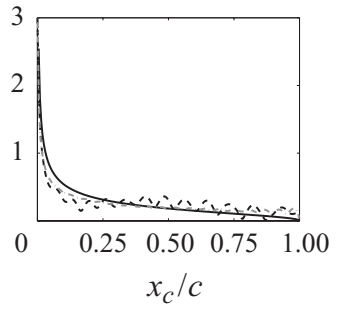

FIGURE 9. Amplitude of the unsteady blade loading $\Delta p_{0}$ along the blade chord, made non-dimensional by the factor $\rho_{0} w_{0} c_{0}: \mathscr{L}=\Delta p_{0} /\left(\rho_{0} w_{0} c_{0}\right)$ from the analytical formulation of $\S 2$, with $\sigma=\pi / 2, \bar{d}=0.98$ and $\chi=35^{\circ}$ (- - -), from Amiet's response function (-. - ) and Sear's response function (-).

pressure contours around the blades are then compared with those obtained from Smith's two-dimensional analysis (Smith 1973), in the manner described by Goldstein (1976). The test case summarized in table 4 was proposed by Hanson and is reported in Dahl's benchmark test case for rotor-stator interaction in category 4 of the Third Computational Aeroacoustic benchmark (Hardin, Huff \& Tam 2000; Namba \& Schulten 2000). The investigated blade row is a stator with $B_{S}=24$ flat-plate vanes of constant chord length $c$ with zero-stagger angle, placed in a straight duct of inner $R_{H}$ and outer $R_{T}$ radii. The steady flow is assumed axial (no swirl) with a constant Mach 
Upon a uniformly valid analytical rectilinear cascade response function

\begin{tabular}{ccccccc}
\hline$R_{H} / R_{T}$ & $B_{S}$ & $B_{R}$ & $\chi$ (deg.) & $\varphi($ deg. $)$ & $\psi($ deg. $)$ & $c$ \\
0.98 & 24 & 16 & 0 & 0 & 0 & $2 \pi R_{T} / B_{S}$ \\
$M_{x d}$ & $M_{T}$ & $\bar{\omega}_{e x}$ & $p$ & $k z_{c 0}$ & $q$ & $V_{p}$ \\
0.5 & 0.6495 & $p B_{R} M_{T} c / R_{T}$ & 1 & $2 \pi q /\left(R_{T}-R_{H}\right)$ & 0 & 0.1
\end{tabular}

TABLE 4. Definition of the stator geometry and vortical excitation parameters.

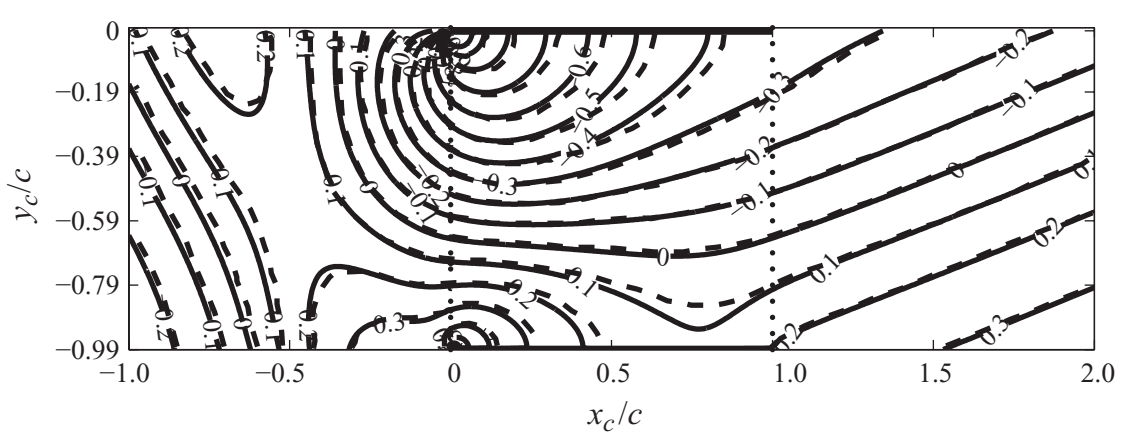

FIGURE 10 . The negative of the imaginary part of the unsteady pressure field at mid-span $\left(R_{m}\right)$ made non-dimensional by the factor $\rho_{0} V_{p} U_{x d}^{2}$ for the test case defined in table 4 obtained with the current model (solid lines) and with Smith's linear two-dimensional model (dashed lines) from Prasad \& Verdon (2002). The minus sign of the unsteady pressure field is plotted due to a convention for the time Fourier transform different from the one presented by Prasad \& Verdon (2002). The contours are plotted with a 0.1 step.

number, $M=0.5$. The incident vortical excitation is defined in the duct cylindrical coordinates $\left(r, \theta_{d}, x_{d}\right)$ as

$$
\begin{aligned}
v\left(r, \theta_{d}, x_{d}, t\right)=U_{x d} \sum_{p=0}^{\infty} & V_{p} \exp \left(\mathrm { i } \left[\left(p B_{R} \Omega x_{d} / U_{d}\right)\right.\right. \\
& \left.\left.+p B_{R} \theta_{d}+2 \pi q\left(r-R_{H}\right) /\left(R_{T}-R_{H}\right)\right]-\mathrm{i} p B_{R} \Omega t\right)
\end{aligned}
$$

with typically $p=1, \Omega=M_{T} c_{0} / R_{T}$, with $M_{T}$ being the tangential Mach number at the upstream rotor blade tip and $\sigma=2 \pi B_{R} / B_{S}$. The pressure field is calculated here in a narrow-annulus configuration with $R_{H} / R_{T}=0.98$ at mid-span $\left(R_{m}\right)$ for a two-dimensional gust $(q=0)$. The contours of the imaginary part of the pressure are plotted in figure 10. The thick horizontal straight lines feature the stator vanes and the dotted vertical lines indicate the boundaries of the inter-vane channel. The present rectilinear model matches Smith's results very well. The current full exact formulation, dealing with both two-dimensional and three-dimensional gusts, could then be used as a reference for future assessment of numerical solvers, of linearized Euler equations, for instance, in rectilinear or narrow-annulus configurations.

\section{Pressure field computed from the blade loading}

According to the acoustic analogy, each blade acts as a chordwise distribution of dipoles, the strength of which is defined by the pressure jump $\Delta p_{0}$. The final aim of this study is to lay the basis for a method, described in detail by Posson et al. (2010), to deal with the three-dimensional geometry of an actual fan stage while resorting to a rectilinear cascade response. The unsteady loading along the blade calculated 

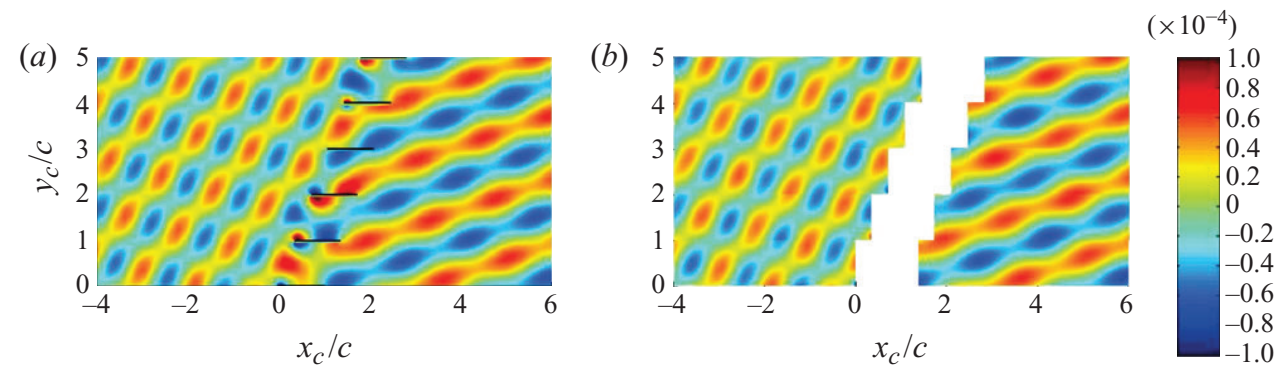

FIGURE 11. Real part of the two-dimensional predicted acoustic pressure made non-dimensional by the factor $\rho_{0} c_{0}^{2}$ for an incident vortical gust of amplitude $w_{0}=0.01 U_{c}$ on a cascade with $\Gamma=1 / \bar{h}=1, \chi=20^{\circ}, M=0.2, \bar{\omega}_{e x}=5, \bar{k}_{z_{c 0}}=0$ and $\sigma=3 \pi / 4:$ (a) complete acoustic field from $(2.2) ;(b)$ external acoustic field computed from the cascade pressure jump and with $B_{S}=40$ blades.

on each strip will be used as a distribution of equivalent dipole sources in the sense of an acoustic analogy. The total field reconstructed by linear superposition of the contributions of all blades assumed to radiate in free space is verified to be identical to Glegg's solution outside the cascade in a two-dimensional configuration. Such a computation is not needed in a rectilinear cascade since the model produces directly the radiated field inside and outside the blades, but, this verification is an important step in the development of a model for the prediction of the broadband noise of an annular fan resorting to a strip theory.

\subsection{Total field reconstruction from the acoustic analogy}

The elementary field of a dipole is deduced from the two-dimensional Green's function for the convected Helmholtz equation with uniform flow, written here as the complex conjugate of Lockard's expression (Lockard 2000) by virtue of a different convention for the time Fourier transform. It is derived from the two-dimensional Green's function in free field with no mean flow using a Prandtl-Glauert transformation:

$$
G(x, y \mid \xi, \eta)=\frac{\mathrm{i}}{4 \beta} \exp \left(-\mathrm{i} M_{t} k \check{x} / \beta^{2}\right) H_{0}^{(2)}\left(\frac{k}{\beta^{2}} \sqrt{\check{x}^{2}+\beta^{2} \check{y}}\right) .
$$

In the present case, the total mean Mach number is $M_{t}=M$, the acoustic wavenumber $k=\omega_{e x} / c_{0}, \check{x}=(x-\xi)$ and $\check{y}=(y-\eta)$ for a source point at $(\xi, \eta)$ and an observer point at $(x, y)$. Strictly speaking, the periodicity of the cascade cannot be reproduced fully because the sum can only be made on a limited number of blades $B_{S}$. Therefore, the result in the vicinity of a reference channel $y_{c} \in\left[0, h\right.$ [ is reliable if $B_{S}$ is large and if the blades are distributed almost symmetrically on each side of this channel. A satisfactory periodic behaviour is achieved around the mid-cascade channel with $B_{S}=40$ and the map covering five channels is then obtained using the inter-blade phase relationship. The maps of figures $11(a)$ and 11(b) agree quite well in terms of wave patterns and amplitudes. Instantaneous pressure profiles obtained by cutting the maps on lines of constant $y_{c}$-values are plotted in figures $12(a)$ and $12(b)$ for a more quantitative comparison with $B_{S}=1000$. The discrepancies reach only $6 \%$ at most at the extrema of the profiles because of the truncation errors. The overall agreement is thus very good, especially when dealing with the intended application to broadband-noise evaluations in decibels. This confirms that the acoustic pressure jump equivalently enables realistic sound predictions based on the acoustic analogy. 

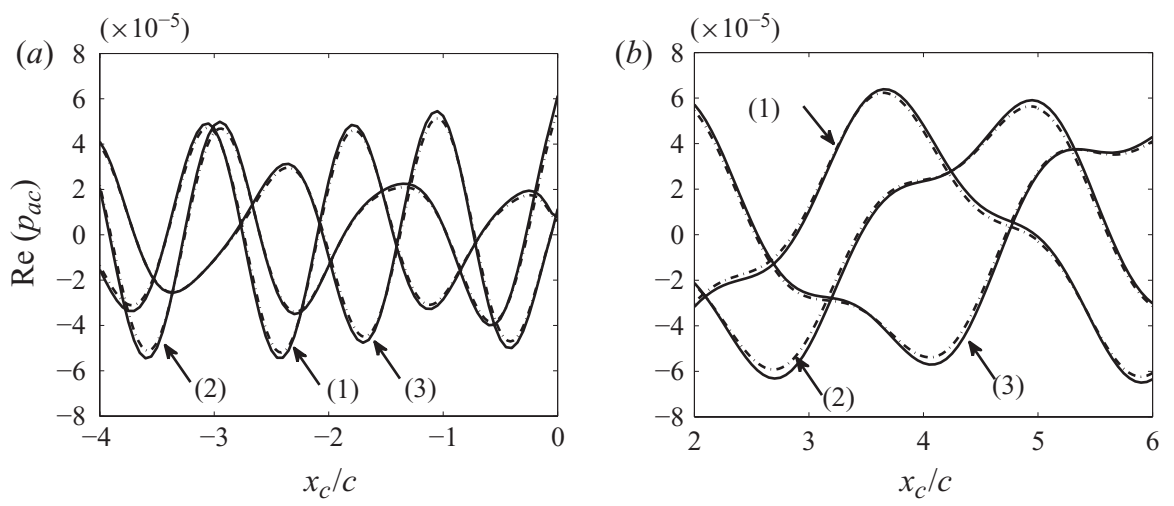

Figure 12. Pressure profiles made non-dimensional by the factor $\rho_{0} c_{0}^{2}$ for an incident vortical gust of amplitude $w_{0}=0.01 U_{c}$ on a cascade with $\Gamma=1, \chi=20^{\circ}, M=0.2, \bar{\omega}_{e x}=5, \bar{k}_{z c 0}=0$, and $\sigma=3 \pi / 4$ at $y_{c}=0.2 c\left(\right.$ curves (1)), $y_{c}=0.5 c$ (curves (2)) and $y_{c}=0.9 c$ (curves (3)) $\left(x_{c} \in[-4,0]\right)$ predicted from $(2.2)(-)$ and reconstructed from the pressure jump on $B_{S}=1000$ blades (-. - .): (a) upstream of the cascade; (b) downstream of the cascade.

It can be used within a strip-theory approach of three-dimensional blade rows of arbitrary geometry, for fan-noise applications. In this case, the artificial condition of an infinite set of blades is replaced by the exact periodicity condition in cylindrical coordinates; the calculation will then be simpler and less prone to numerical errors. The remaining issue will then be the ability of the linear-cascade response function to simulate the behaviour of the unsteady blade loading of a three-dimensional annular cascade. First results by Posson \& Roger (2007) have shown the accuracy of the approach for high hub-to-tip ratio configurations. The discrepancies observed for low hub-to-tip ratio have required some modifications of the model to introduce the actual annular effect on the wave equation. The proposed correction is detailed by Posson et al. (2010). They concluded that the correction is needed to get results closer to the actual annular geometry, but discrepancies are still found in the rather low-frequency harmonic test case considered in this reference.

\subsection{Comparison with an isolated-airfoil response function}

This section addresses the ability of the isolated-airfoil response function to reproduce the acoustic field of a rectilinear blade row. This is achieved by replacing the cascade pressure jump in the computations of $\S 5.1$ by Amiet's expression and by enforcing the same inter-blade phase angle $\sigma$ between the blades. Comparing the results in figure 13 for the test case of $\S 5.1$ shows that the interference patterns are qualitatively similar. This is expected since the correct phase shift is imposed. However, large errors are observed on the local amplitude of the pressure field, typically overpredicted by at most $43 \%$ downstream and underpredicted upstream by at most $55 \%$, with a mean relative error of $30 \%$.

The results of a second test case, defined in table 5 , with a small solidity $\Gamma=0.45$ and a small overlap $1-\bar{d}=0.20$ (instead of 0.64 ) are shown in figures 14 and 15 for two incident gusts with different inter-blade phase angles. The two cascade response functions for the unsteady blade loading are found to be close to the isolatedairfoil response function in figure 14. Yet varying the inter-blade phase angle notably modifies the response. The difference between the two cascade responses is of the same order of magnitude as the difference between the isolated-airfoil and cascade responses. 


\begin{tabular}{cccccccc}
\hline Sub-case & $\bar{h}$ & $\chi$ (deg.) & $M$ & $\bar{\omega}_{\text {ex }}$ & $\sigma$ & $\bar{k}_{z_{c 0}}$ & $\varphi$ \\
(I) & 2.2 & 20 & 0.2 & 5 & $\pi / 2$ & 0 & 0 \\
(II) & 2.2 & 20 & 0.2 & 5 & $3 \pi / 4$ & 0 & 0
\end{tabular}

TABLE 5. Input parameters of the second case to compare the isolated-airfoil and the cascade response functions when the overlap tends to zero (used in figure 15).
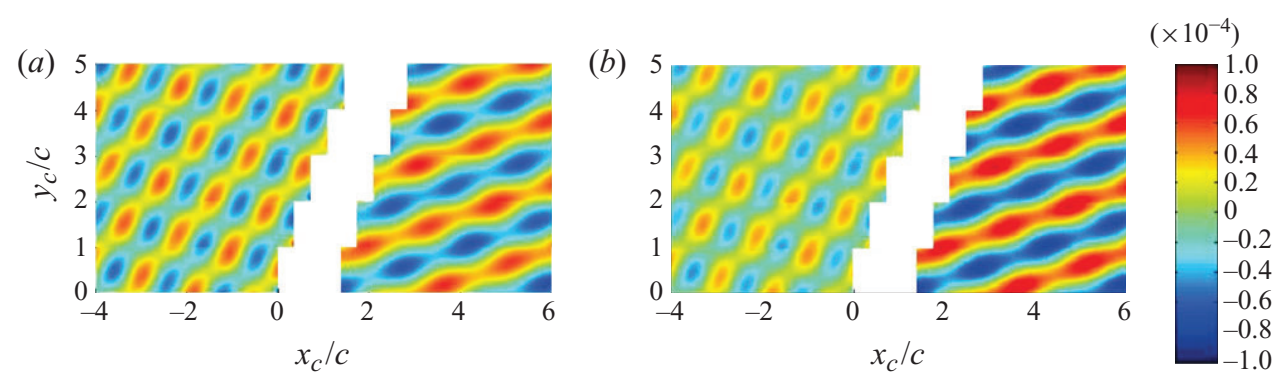

FIGURE 13. Real part of the two-dimensional predicted acoustic pressure made non-dimensional by the factor $\rho_{0} c_{0}^{2}$ for an incident vortical gust of amplitude $w_{0}=0.01 U_{c}$ on a cascade with $\Gamma=1, \chi=20^{\circ}, M=0.2, \bar{\omega}_{e x}=5, \bar{k}_{z_{c 0}}=0$, and $\sigma=3 \pi / 4$. (a) External acoustic field computed from the cascade pressure jump and with $B_{S}=40$ blades ; $(b)$ external acoustic field computed from the isolated-airfoil pressure jump and $B_{S}=40$ blades phased-shifted by $\sigma$.

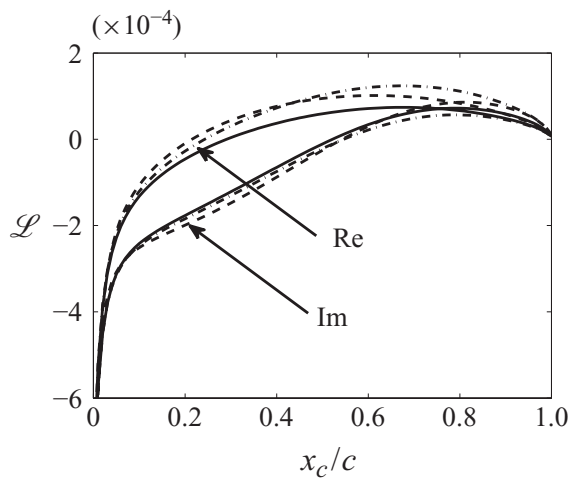

Figure 14. Chordwise distributions of the real and imaginary parts of the unsteady blade loading $\Delta p_{0}$ made non-dimensional by the factor $\rho_{0} w_{0} c_{0}: \mathscr{L}=\Delta p_{0} /\left(\rho_{0} w_{0} c_{0}\right)$ for cases defined in table 5 from Amiet's response function (- - $)$ and from the analytical formulation of $\S 2$ in sub-case (I) $(\sigma=\pi / 2)(-\cdot-)$ and in sub-case (II) $(\sigma=3 \pi / 4)(-)$.

A pressure profile along the line $y_{c} / c=3.025$ is plotted in figure 15 for a quantitative comparison of the radiated fields. First in case (I) (figure 15a), the solution obtained with the isolated-airfoil response function is in good agreement with the cascade solution. The cascade behaves consistently as the blade overlap tends to zero. The more different patterns of case (II) (figure 15b) illustrate the dominant effect of the inter-blade phase angle. But again the agreement of the pressure profiles in figure 15(b) shows that the field is rather well reproduced using the single-airfoil theory despite some discrepancies in amplitudes. As the relative blade-to-blade distance increases, the isolated-airfoil response function can be used as an alternative whatever the 

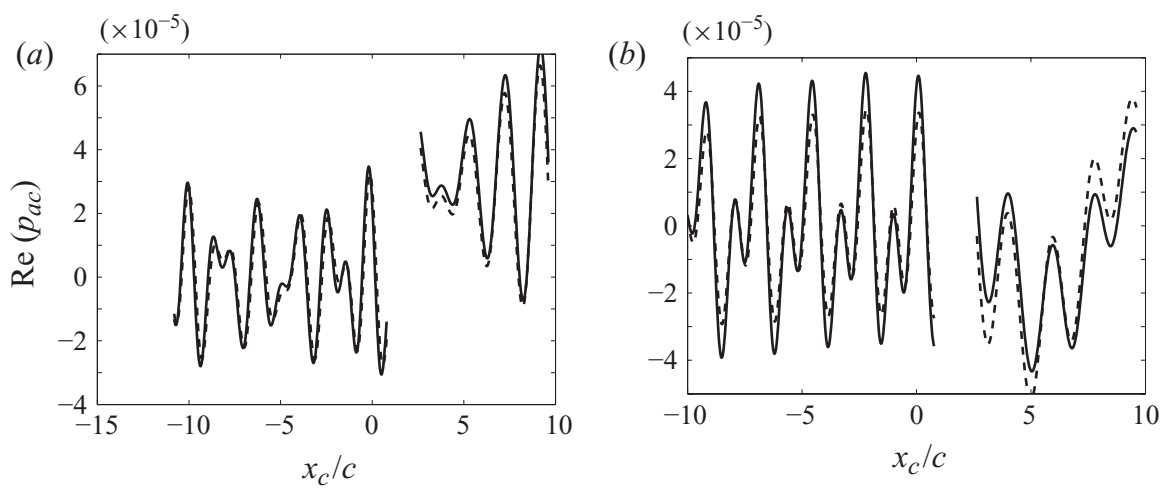

FIGURE 15. Pressure profiles at $y / c=3.025$ made non-dimensional by the factor $\rho_{0} c_{0}^{2}$ for an incident vortical gust of amplitude $w_{0}=0.01 U_{c}$ on a cascade with $\Gamma=0.45, \chi=20^{\circ}, M=0.2$, $\bar{\omega}_{e x}=5, \bar{k}_{z_{c 0}}=0$ and $(a) \sigma=\pi / 2 ;(b) \sigma=3 \pi / 4$. External acoustic field computed from the cascade pressure jump and with $B_{S}=1000$ blades $(-)$ and external acoustic field computed from the isolated-airfoil pressure jump and $B_{S}=40$ blades phased-shifted by $\sigma($ - $)$.

inter-blade phase angle might be. It should be noticed that $\sigma=\pi / 2$ plays a particular role as the corresponding gust amplitude is zero on a blade when it reaches its maximum at the neighbouring blade. On the contrary, when the inter-blade phase angle is 0 or $\pi$, the gust amplitudes are, respectively, in phase or out of phase, introducing large interferences. The configuration $\sigma=\pi / 2$ is therefore a particular case where interferences are minimized and the cascade blades behave closer to an isolated airfoil. The neighbouring blade 'acts' as a 'ghost' and the effective solidity is halved.

\section{Concluding remarks}

Glegg's model of a rectilinear cascade response has been re-addressed and a full analytical solution has been derived for the acoustic field in the whole space, including the inter-blade channels, and for the unsteady loading on the blades, based on an extensive application of the residue theorem. The extended analytical solution has been successfully compared to alternative rectilinear cascade models both in terms of unsteady loading on blades and pressure field in the blade passage.

The unsteady blade loading has also been used as an equivalent source in the sense of an acoustic analogy in a rectilinear configuration. The results confirm that it ensures reliable sound predictions. Unsteady blade loadings could then be used within a strip-theory approach for three-dimensional blade rows of arbitrary geometry for fan-noise applications.

The unsteady blade loading and the radiated acoustic pressure field produced by the gust-cascade interaction have been compared with a classical isolated-airfoil solution, showing large differences in both quantities due to the cascade effect when the blade overlap is significant. Nevertheless, when the blade overlap goes to zero with a small or moderate solidity, the unsteady blade loading gets closer to the isolated-airfoil response. This suggests that both models can be used as a continuation of each other if needed.

The authors wish to acknowledge Snecma which supported the CIFRE PhD thesis during which most of this study was conducted. They would also like to acknowledge 
GAUS, the acoustic groups of l'Universite de Sherbrooke (QC, Canada) for supporting the continuation of this work.

\section{Appendix A. Analytical model formulation}

\section{A.1. Reminder of main parameters of Glegg's cascade model}

For convenience, the same quantities and notations as in Glegg's (1999) model are introduced and recalled:

$$
\begin{aligned}
& M=\frac{U_{c}}{c_{0}}, \quad \beta=\sqrt{1-M^{2}}, \quad \omega=\omega_{e x}-k_{z_{c 0}} W_{c}, \\
& s=\sqrt{d^{2}+h^{2}}, \quad s_{e}=\sqrt{d^{2}+\beta^{2} h^{2}}, \quad \tan \chi_{e}=\frac{d}{\beta h}, \\
& \kappa=\omega /\left(c_{0} \beta^{2}\right), \quad \kappa_{e}^{2}=\kappa^{2}-\left(k_{z_{c 0}} / \beta\right)^{2}, \quad \xi=k_{x_{c}}-\kappa M, \\
& \delta_{l}=\kappa M-\vartheta_{l-1}, \quad \varepsilon_{l}=\kappa M+\vartheta_{l-1}, \quad \vartheta_{l}=\sqrt{\kappa_{e}^{2}-\left(\frac{l \pi}{\beta h}\right)^{2}} \\
& \sigma=k_{x_{c}} d+k_{y_{c}} h, \quad f_{q}=\frac{\sigma+\kappa M d-2 \pi q}{\sqrt{d^{2}+\beta^{2} h^{2}}}, \quad \eta_{q}^{ \pm}=-f_{q} \sin \chi_{e} \pm \cos \chi_{e} \sqrt{\kappa_{e}^{2}-f_{q}^{2}}, \\
& \lambda_{q}^{ \pm}=\kappa M+\eta_{q}^{ \pm}, \quad \zeta_{q}^{ \pm}=\zeta\left(\lambda_{q}^{ \pm}\right), \quad \zeta\left(k_{x_{c 0}}\right)=\sqrt{\left(\omega+k_{x_{c}} U_{c}\right)^{2} / c_{0}^{2}-k_{x_{c}}^{2}-k_{z_{c}}^{2}}, \\
& \rho=\sigma+\kappa M d, \quad d_{n}=\left\{\begin{array}{ll}
2, & \text { if } n=0, \\
1, & \text { else, }
\end{array} \quad Z=-\frac{\mathrm{i} \xi}{\pi}\left[\beta h \log \left(2 \cos \chi_{e}\right)+\chi_{e} d\right] .\right.
\end{aligned}
$$

A new variable $\mathscr{S}_{q}^{ \pm}$taking the values +1 and -1 is defined as follows, ensuring for all integer $q, \zeta_{q}^{ \pm} h=\mathscr{S} \frac{ \pm}{q}\left(\lambda \frac{ \pm}{q} d+\sigma-2 \pi q\right)$ :

$$
\begin{aligned}
& \mathscr{S}_{q}^{+}=\left\{\begin{array}{ll}
-1, & \text { if } \zeta_{q}^{+} h=-\left[\sigma+\lambda_{q}^{+} d-2 \pi q\right], \\
+1, & \text { if } \zeta_{q}^{+} h=+\left[\sigma+\lambda_{q}^{+} d-2 \pi q\right],
\end{array}\right\} \\
& \mathscr{S}_{q}^{-}=\left\{\begin{array}{ll}
-1, & \text { if } \zeta_{q}^{-} h=-\left[\sigma+\lambda_{q}^{-} d-2 \pi q\right], \\
+1, & \text { if } \zeta_{q}^{-} h=+\left[\sigma+\lambda_{q}^{-} d-2 \pi q\right] .
\end{array}\right\}
\end{aligned}
$$

The function $j$, defined as

$$
j\left(k_{x_{c}}\right)=\frac{\zeta \sin \zeta h}{4 \pi(\cos \zeta h-\cos \xi d+\rho)},
$$

is split into the product of an analytical function $J_{+}$in the upper half-plane and an analytical function $J_{-}$in the lower half-plane, with

$$
J_{+}\left(k_{x_{c}}\right)=\frac{\beta \kappa_{e} \sin \left(\beta \kappa_{e} h\right) \mathrm{e}^{Z}}{4 \pi\left(\cos \left(\beta \kappa_{e} h\right)-\cos (\rho)\right)} \frac{\prod_{l=0}^{\infty}\left(1-\xi / \theta_{l}\right)}{\prod_{q=-\infty}^{\infty}\left(1-\xi / \eta_{q}^{-}\right)}, \quad J_{-}\left(k_{x_{c}}\right)=\frac{\mathrm{e}^{-Z} \prod_{l=0}^{\infty}\left(1-\xi / \vartheta_{l}\right)}{\prod_{q=-\infty}^{\infty}\left(1-\xi / \eta_{q}^{+}\right)} .
$$

The variables $\delta_{l}$ and $\varepsilon_{l}$, for all $l \geqslant 1$, are the inter-blade channel wavenumbers, zeros of $J_{+}$and $J_{-}$, respectively. The four terms $D^{(j)}$ of the Fourier transform of the jump 
of the velocity potential read

$$
\begin{aligned}
D^{(1)}\left(k_{x_{c}}\right) & =\frac{-\mathrm{i} w_{0}}{(2 \pi)^{2}\left(k_{x_{c}}+k_{x_{c} 0}\right) J_{-}\left(-k_{x_{c} 0}\right) J_{+}\left(k_{x_{c}}\right)}, \\
D^{(2)}\left(k_{x_{c}}\right) & =-\sum_{n \geqslant 1} \frac{A_{n} \exp \left(\mathrm{i}\left(k_{x_{c}}-\delta_{n}\right) c\right) J_{-}\left(\delta_{n}\right)}{\mathrm{i}\left(\omega_{g}+k_{x_{c}} U_{c}\right)\left(k_{x_{c}}-\delta_{n}\right) J_{-}\left(k_{x_{c}}\right)}, \\
D^{(3)}\left(k_{x_{c}}\right) & =-\sum_{l=1}^{\infty} \frac{B_{l} J_{+}\left(\varepsilon_{l}\right)}{\left(k_{x_{c}}-\varepsilon_{l}\right) J_{+}\left(k_{x_{c}}\right)}, \\
D^{(4)}\left(k_{x_{c}}\right) & =-\sum_{n=1}^{\infty} \frac{C_{n} \mathrm{e}^{\mathrm{i}\left(k_{x_{c}}-\delta_{n}\right) c} J_{-}\left(\delta_{n}\right)}{\mathrm{i}\left(\omega_{g}+k_{x_{c}} U_{c}\right)\left(k_{x_{c}}-\delta_{n}\right) J_{-}\left(k_{x_{c}}\right)},
\end{aligned}
$$

with ((A.26) of the reference paper)

$$
A_{n}=\frac{w_{0}\left(\omega_{g}+\delta_{n} U_{c}\right)}{(2 \pi)^{2}\left(\delta_{n}+k_{x_{c 0}}\right) J_{+}^{\prime}\left(\delta_{n}\right) J_{-}\left(-k_{x_{c 0}}\right)}, \quad n \geqslant 1 .
$$

The value of $A_{0}$ for sound generation is in fact zero by definition of $k_{x_{c 0}}$ and $\omega_{g}$. The coefficients $B_{n}$ and $C_{n}$ are also obtained as in Glegg's model by

$$
\left.\begin{array}{l}
B=F \cdot(A+C) \\
C=L \cdot B
\end{array}\right\}
$$

where $\boldsymbol{A}=\left(A_{n}\right)_{n \in \mathbb{N}}, \boldsymbol{F}=\left(F_{l, n}\right)_{(l, n) \in \mathbb{N}^{* 2}}, \boldsymbol{L}=\left(L_{l, n}\right)_{(l, n) \in \mathbb{N}^{* 2}}$ with the same definition of $F_{l, n}$ and $L_{l, n}$ as in (A.48) and (A.49) of the reference paper:

$$
F_{l, n}=-\frac{J_{-}\left(\delta_{n}\right) \exp \left(\mathrm{i}\left(\varepsilon_{l}-\delta_{n}\right) c\right)}{\mathrm{i}\left(\omega_{g}+\varepsilon_{l} U_{c}\right)\left(\varepsilon_{l}-\delta_{n}\right) J_{-}^{\prime}\left(\varepsilon_{l}\right)}, \quad L_{l, n}=\frac{\mathrm{i}\left(\omega_{g}+\delta_{l} U_{c}\right) J_{+}\left(\varepsilon_{n}\right)}{\left(\varepsilon_{n}-\delta_{l}\right) J_{+}^{\prime}\left(\delta_{l}\right)} .
$$

Then $D\left(\lambda_{q}^{+}\right)$is given by

$$
\begin{aligned}
D\left(\lambda_{q}^{+}\right) & =\frac{-\mathrm{i} w_{0}}{(2 \pi)^{2}\left(\lambda_{q}^{+}+k_{x_{c 0}}\right) J_{+}\left(\lambda_{q}^{+}\right) J_{-}\left(-k_{x_{c 0}}\right)}-\sum_{l=1}^{\infty} \frac{B_{l} J_{+}\left(\varepsilon_{l}\right)}{\left(\lambda_{q}^{+}-\varepsilon_{l}\right) J_{+}\left(\lambda_{q}^{+}\right)} \\
& =D^{(1,3)}\left(\lambda_{q}^{+}\right), \quad \forall q \in \mathbb{Z},
\end{aligned}
$$

and $D\left(\lambda_{q}^{-}\right)$by

$$
D\left(\lambda_{q}^{-}\right)=-\sum_{n=1}^{\infty} \frac{\left(A_{n}+C_{n}\right) \exp \left(\mathrm{i}\left(\lambda_{q}^{-}-\delta_{n}\right) c\right) J_{-}\left(\delta_{n}\right)}{\mathrm{i}\left(\omega+\lambda_{q}^{-} U_{c c}\right)\left(\lambda_{q}^{-}-\delta_{n}\right) J_{-}\left(\lambda_{q}^{-}\right)}=D^{(2,4)}\left(\lambda_{q}^{-}\right), \quad \forall q \in \mathbb{Z} .
$$

\section{A.2. Acoustic potential in the inter-blade channel}

A general form of the acoustic part $\phi_{a c}$ of the scattered velocity potential $\phi$ is derived by application of the residue theorem following the method presented in $\S 2$. The pressure-free part of the potential convected by the mean flow, $\phi_{v}$, can be obtained in a similar way but is not given here for the sake of conciseness. 
In patch (a) $\left(0 \leqslant x_{c} \leqslant d\right)$ :

$$
\begin{aligned}
\phi_{a c, a}\left(x_{c}, y_{c}\right) & =\sum_{q \in \mathbb{Z}} \phi_{a c, a, 1, \lambda, q}^{+}\left(x_{c}, y_{c}\right)+\sum_{l \geqslant 1} \phi_{a c, a, 1, l}^{+}\left(x_{c}, y_{c}\right) \\
+ & \sum_{n \geqslant 1}\left(\phi_{a c, a, 2, n}^{+}\left(x_{c}, y_{c}\right)+\sum_{l \geqslant 1}\left[\phi_{a c, a, 2, n, l}^{+}\left(x_{c}, y_{c}\right)+\phi_{a c, a b, n, l}^{-}\left(x_{c}, y_{c}\right)\right]\right) .
\end{aligned}
$$

In patch (b) $\left(d<x_{c}<c\right)$ :

$$
\phi_{a c, b}\left(x_{c}, y_{c}\right)=\sum_{n \geqslant 1} \phi_{a c, b c, n}^{+}\left(x_{c}, y_{c}\right)+\sum_{n \geqslant 1} \sum_{l \geqslant 1}\left[\phi_{a c, b c, n, l}^{+}\left(x_{c}, y_{c}\right)+\phi_{a c, a b, n, l}^{-}\left(x_{c}, y_{c}\right)\right] \text {. }
$$

In patch $(\mathrm{c})\left(c \leqslant x_{c} \leqslant c+d\right)$ :

$$
\begin{array}{r}
\phi_{a c, c}\left(x_{c}, y_{c}\right)=\sum_{n \geqslant 1}\left[\phi_{a c, b c, n}^{+}\left(x_{c}, y_{c}\right)+\phi_{a c, c, 2, n}^{-}\left(x_{c}, y_{c}\right)\right]+\sum_{q \in \mathbb{Z}} \phi_{a c, c, 2, \lambda, q}^{-}\left(x_{c}, y_{c}\right) \\
+\sum_{n \geqslant 1} \sum_{l \geqslant 1}\left[\phi_{a c, b c, n, l}^{+}\left(x_{c}, y_{c}\right)+\phi_{a c, c, 1, n, l}^{-}\left(x_{c}, y_{c}\right)\right]
\end{array}
$$

The different terms of the sums are defined as

$$
\left.\begin{array}{rl}
\phi_{a c, a, 1, \lambda, q}^{+}\left(x_{c}, y_{c}\right)= & -\frac{\mathrm{i} \pi \zeta_{q}^{+} D\left(\lambda_{q}^{+}\right) \cos \left(\zeta_{q}^{+} y_{c}\right) \exp \left(\mathrm{i}\left(\sigma+\lambda_{q}^{+} d\right)\right) \exp \left(-\mathrm{i} \lambda_{q}^{+} x_{c}\right)}{\sin \left(\lambda_{q}^{+} d+\sigma\right)\left(\zeta_{q}^{+} d+S_{q}^{+} h \beta^{2} \eta_{q}^{+}\right)}, \\
\phi_{a c, a, 1, l}^{+}\left(x_{c}, y_{c}\right)= & +\frac{\mathrm{i} \pi B_{l} \cos \left(\zeta\left(\varepsilon_{l}\right) y_{c}\right) \exp \left(\mathrm{i}\left(\varepsilon_{l}\left(d-x_{c}\right)+\sigma\right)\right)}{\left(\cos \left(\zeta\left(\varepsilon_{l}\right) h\right)-\cos \left(\varepsilon_{l} d+\sigma\right)\right)}, \\
\phi_{a c, a, 2, n}^{+}\left(x_{c}, y_{c}\right)= & -\frac{w_{0} J_{-}\left(\delta_{n}\right) \cos \left(\zeta\left(\delta_{n}\right) y_{c}\right) \mathrm{e}^{-\mathrm{i} \delta_{n} x_{c}}}{J_{-}\left(-k_{x_{c} 0}\right)\left(\delta_{n}+k_{x_{c} 0}\right) \beta^{2} h d_{n} \vartheta_{n-1}}, \\
\phi_{a c, a, 2, n, l}^{+}\left(x_{c}, y_{c}\right)= & \frac{(2 \pi)^{2} \mathrm{i} B_{l} J_{+}\left(\varepsilon_{l}\right) J_{-}\left(\delta_{n}\right) \cos \left(\zeta\left(\delta_{n}\right) y_{c}\right) \mathrm{e}^{-\mathrm{i} \delta_{n} x_{c}}}{\left(\delta_{n}-\varepsilon_{l}\right) \beta^{2} h d_{n} \vartheta_{n-1}}, \\
\phi_{a c, a b, n, l}^{-}\left(x_{c}, y_{c}\right)= & \frac{(2 \pi)^{2}\left(A_{n}+C_{n}\right) J_{-}\left(\delta_{n}\right) J_{+}\left(\varepsilon_{l}\right) \exp \left(\mathrm{i} \varepsilon_{l}\left(c-x_{c}\right)-\mathrm{i} \delta_{n} c\right)}{\left.\beta_{l} U_{c}\right)\left(\varepsilon_{l}-\delta_{n}\right)} \\
& \times \frac{\left[\cos \left(\zeta\left(\varepsilon_{l}\right) y_{c}\right) \exp \left(\mathrm{i}\left(\varepsilon_{l} d+\sigma\right)\right)-\cos \left(\zeta\left(\varepsilon_{l}\right)\left(y_{c}-h\right)\right)\right]}{\beta^{2} h \theta_{l-1}(-1)^{l-1} d_{l}(-1)^{n-1} d_{n}}, \\
\phi_{a c, b c, n}^{+}\left(x_{c}, y_{c}\right)= & \frac{w_{0} J_{-}\left(\delta_{n}\right)\left[\cos \left(\zeta\left(\delta_{n}\right) y_{c}\right) \exp \left(\mathrm{i}\left(\sigma+\delta_{n} d\right)\right)-\cos \left(\zeta\left(\delta_{n}\right)\left(y_{c}-h\right)\right)\right] \mathrm{e}^{-\mathrm{i} \delta_{n} x_{c}}}{J_{-}\left(-k_{x_{c} 0}\right)\left(\delta_{n}+k_{x_{c} 0}\right) \beta^{2} h \vartheta_{n-1}(-1)^{n-1} d_{n}} \\
\phi_{a c, b c, n, l}^{+}\left(x_{c}, y_{c}\right)= & -\frac{(2 \pi)^{2} \mathrm{i} B_{l} J_{+}\left(\varepsilon_{l}\right)}{\left(\delta_{n}-\varepsilon_{l}\right)} \\
& \times \frac{J_{-}\left(\delta_{n}\right)\left[\cos \left(\zeta\left(\delta_{n}\right) y_{c}\right) \exp \left(\mathrm{i}\left(\sigma+\delta_{n} d\right)\right)-\cos \left(\zeta\left(\delta_{n}\right)\left(y_{c}-h\right)\right)\right] \mathrm{e}^{-\mathrm{i} \delta_{n} x_{c}}}{\beta^{2}},
\end{array}\right\}
$$




$$
\left.\begin{array}{rl}
\phi_{a c, c, 2, n}^{-}\left(x_{c}, y_{c}\right) & =+\frac{\pi\left(A_{n}+C_{n}\right) \cos \left(\zeta\left(\delta_{n}\right)\left(y_{c}-h\right)\right) \mathrm{e}^{-\mathrm{i} \delta_{n} x_{c}}}{\left(\omega+\delta_{n} U_{c}\right)\left(\cos \left(\zeta\left(\delta_{n}\right) h\right)-\cos \left(\delta_{n} d+\sigma\right)\right)}, \\
\phi_{a c, c, 1, n, l}^{-}\left(x_{c}, y_{c}\right) & =\frac{(2 \pi)^{2}\left(A_{n}+C_{n}\right) J_{-}\left(\delta_{n}\right) J_{+}\left(\varepsilon_{l}\right) \cos \left(\zeta\left(\varepsilon_{l}\right) y_{c}\right) \exp \left(\mathrm{i} \varepsilon_{l}\left(c+d-x_{c}\right)-\mathrm{i} \delta_{n} c+\mathrm{i} \sigma\right)}{\left(\omega+\varepsilon_{l} U_{c}\right)\left(\varepsilon_{l}-\delta_{n}\right) \beta^{2} h \theta_{l-1}(-1)^{l-1} d_{l}}, \\
\phi_{a c, c, 2, \lambda, q}^{-}\left(x_{c}, y_{c}\right) & =-\frac{\mathrm{i} \pi D^{(2,4)}\left(\lambda_{q}^{-}\right) \zeta_{q}^{-} \cos \left(\zeta_{q}^{-}\left(y_{c}-h\right)\right) \mathrm{e}^{-\mathrm{i} \lambda_{q}^{-} x_{c}}}{\sin \left(\lambda_{q}^{-} d+\sigma\right)\left(\zeta_{q}^{-} d+S_{q}^{-} h \beta^{2} \eta_{q}^{-}\right)} .
\end{array}\right\}
$$

\section{A.3. Pressure jump on the blade}

The expression for the pressure jump can then be derived from (2.13) and (2.16) using the expressions of the acoustic potential (see (A 14)-(A 18)). It is then written as follows, in terms of the factors listed below.

On segment I $\left(0<x_{c}<\min (c-d, d)\right)$ :

$$
\Delta p_{0}\left(x_{c}\right)=(D P 2+D P 3+D P 4+D P 5+D P 6)\left(x_{c}\right) .
$$

If $d<c / 2$ on segment II $\left(d<x_{c}<c-d\right)$ :

$$
\Delta p_{0}\left(x_{c}\right)=(D P 6+D P 8+D P 9)\left(x_{c}\right) .
$$

If $c / 2<d<c$ on segment II' $\left(c-d<x_{c}<d\right)$ :

$$
\Delta p_{0}\left(x_{c}\right)=(D P 2+D P 3+D P 4+D P 5+D P 10+D P 11+D P 12)\left(x_{c}\right) .
$$

On segment III $\left(\max (c-d, d)<x_{c}<c\right)$ :

$$
\Delta p_{0}\left(x_{c}\right)=(D P 8+D P 9+D P 10+D P 11+D P 12)\left(x_{c}\right) .
$$

The $D P j$ terms read

$$
\left.\begin{array}{rl}
D P 2\left(x_{c}\right)= & -\sum_{n \geqslant 1} \frac{\rho_{0} \mathrm{i} w_{0} J_{-}\left(\delta_{n}\right)\left(\omega+\delta_{n} U_{c}\right)\left(2-\exp \left(-\mathrm{i}\left(\sigma+\delta_{n} d+(n-1) \pi\right)\right)\right)}{J_{-}\left(-k_{x_{c}}\right)\left(\delta_{n}+k_{x_{c}}\right) \beta^{2} h d_{n} \vartheta_{n-1}} \mathrm{e}^{-\mathrm{i} \delta_{n} x_{c}}, \\
D P 3\left(x_{c}\right)= & -\sum_{n \geqslant 1} \sum_{l \geqslant 1} \frac{\rho_{0}(2 \pi)^{2} B_{l} J_{+}\left(\varepsilon_{l}\right) J_{-}\left(\delta_{n}\right)\left(\omega+\delta_{n} U_{c}\right)}{\left(\delta_{n}-\varepsilon_{l}\right) \beta^{2} h d_{n} \vartheta_{n-1}} \\
& \times\left(2-\exp \left(-\mathrm{i}\left(\sigma+\delta_{n} d+(n-1) \pi\right)\right)\right) \mathrm{e}^{-\mathrm{i} \delta_{n} x_{c}}, \\
D P 4\left(x_{c}\right)= & +\sum_{q \in \mathbb{Z}} \frac{\rho_{0} \pi \zeta_{q}^{+} D\left(\lambda_{q}^{+}\right) \exp \left(\mathrm{i}\left(\sigma+\lambda_{q}^{+}\left(d-x_{c}\right)\right)\right)\left(\omega+\lambda_{q}^{+} U_{c}\right)}{\sin \left(\lambda_{q}^{+} d+\sigma\right)\left(\zeta_{q}^{+} d+\mathscr{S}_{q}^{+} h \beta^{2} \eta_{q}^{+}\right)}, \\
D P 5\left(x_{c}\right)= & -\sum_{l \geqslant 1} \frac{\rho_{0} \pi B_{l}\left(\omega+\varepsilon_{l} U_{c}\right) \exp \left(\mathrm{i}\left(\varepsilon_{l}\left(d-x_{c}\right)+\sigma\right)\right)}{(-1)^{l-1}-\cos \left(\varepsilon_{l} d+\sigma\right)} \\
D P 6\left(x_{c}\right)= & -\sum_{l \geqslant 1} \sum_{n \geqslant 0} \frac{2 \rho_{0} \mathrm{i}(2 \pi)^{2}\left(A_{n}+C_{n}\right) J_{+}\left(\varepsilon_{l}\right) J_{-}\left(\delta_{n}\right) \exp \left(\mathrm{i}\left(\varepsilon_{l}\left(c-x_{c}\right)-\mathrm{i} \delta_{n} c\right)\right)}{\left(\varepsilon_{l}-\delta_{n}\right)} \\
& \times \frac{1-\cos \left(\sigma+\varepsilon_{l} d+(l-1) \pi\right)}{\beta^{2} h d_{l} \theta_{l-1}},
\end{array}\right\}
$$




$$
\left.\begin{array}{rl}
\operatorname{DP} 8\left(x_{c}\right)= & -\sum_{n \geqslant 1} \frac{2 \rho_{0} \mathrm{i} w_{0}\left(\omega+\delta_{n} U_{c}\right) J_{-}\left(\delta_{n}\right)}{\left(\delta_{n}+k_{x_{c} 0}\right) J_{-}\left(-k_{x_{c}}\right) \beta^{2} h d_{n} \vartheta_{n-1}} \\
& \times\left(1-\cos \left(\sigma+\delta_{n} d+(n-1) \pi\right)\right) \mathrm{e}^{-\mathrm{i} \delta_{n} x_{c}}, \\
\operatorname{DP9}\left(x_{c}\right)= & -\sum_{n \geqslant 1} \sum_{l \geqslant 1} \frac{2 \rho_{0}(2 \pi)^{2} B_{l} J_{+}\left(\varepsilon_{l}\right) J_{-}\left(\delta_{n}\right)\left(\omega+\delta_{n} U_{c}\right)}{\left(\delta_{n}-\varepsilon_{l}\right)} \mathrm{e}^{-\mathrm{i} \delta_{n} x_{c}} \\
& \times \frac{1-\cos \left(\sigma+\delta_{n} d+(n-1) \pi\right)}{\beta^{2} h d_{n} \vartheta_{n-1}}, \\
\operatorname{DP} 10\left(x_{c}\right)= & +\sum_{l \geqslant 1} \sum_{n \geqslant 0} \frac{\rho_{0} \mathrm{i}(2 \pi)^{2}\left(A_{n}+C_{n}\right) J_{+}\left(\varepsilon_{l}\right) J_{-}\left(\delta_{n}\right) \exp \left(\mathrm{i}\left(\varepsilon_{l}\left(c-x_{c}\right)-\mathrm{i} \delta_{n} c\right)\right)}{\left(\varepsilon_{l}-\delta_{n}\right)} \\
& \times \frac{\exp \left(\mathrm{i}\left(\sigma+\varepsilon_{l} d+(l-1) \pi\right)\right)-2}{\beta^{2} h d_{l} \theta_{l-1}}, \\
D P 11\left(x_{c}\right)= & -\sum_{q \in \mathbb{Z}} \frac{\rho_{0} \pi \zeta_{q}^{-} D^{(2,4)}\left(\lambda_{q}^{-}\right) \exp \left(-\mathrm{i}\left(\sigma+\lambda_{q}^{-}\left(d+x_{c}\right)\right)\right)\left(\omega+\lambda_{q}^{-} U_{c}\right)}{\sin \left(\lambda_{q}^{-} d+\sigma\right)\left(\zeta_{q}^{-} d+\mathscr{S}_{q}^{-} h \beta^{2} \eta_{q}^{-}\right)} \\
D P 12\left(x_{c}\right)= & -\sum_{n \geqslant 1} \frac{\rho_{0} \mathrm{i} \pi\left(A_{n}+C_{n}\right) \exp \left(-\mathrm{i}\left(\sigma+\delta_{n}\left(d+x_{c}\right)\right)\right)}{(-1)^{n-1}-\cos \left(\delta_{n} d+\sigma\right)} .
\end{array}\right\}
$$

Appendix B. Particular case: $d=0$ and $\sigma \equiv 0[2 \pi]$

\section{B.1. Recasting of the original model}

In the particular case where blades are not staggered $(d=0)$ and the oncoming gust is in phase on all blades $(\sigma \equiv 0[2 \pi])$, the whole model must be revisited, leading to the following expressions:

$$
j\left(k_{x_{c}}\right)=+\frac{\mathrm{i} \zeta}{4 \pi} \sum_{n=-\infty}^{\infty} \mathrm{e}^{\mathrm{i} \zeta|n h|}=-\frac{\zeta}{4 \pi \tan (\zeta h / 2)} .
$$

The function $j$ is split into the product of an analytical function $J_{+}$in the upper half-plane and an analytical function $J_{-}$in the lower half-plane, with

$$
J_{+}\left(k_{x_{c}}\right)=-\frac{\mathrm{e}^{Z} \beta \kappa_{e} \cos \left(\beta \kappa_{e} h / 2\right)}{4 \pi \sin \left(\beta \kappa_{e} h / 2\right)} \frac{\prod_{l=0}^{+\infty}\left(1-\xi / \theta_{2 l+1}\right)}{\prod_{q=1}^{+\infty}\left(1-\xi / \theta_{2 q}\right)}, \quad J_{-}\left(k_{x_{c}}\right)=\frac{\mathrm{e}^{-Z} \prod_{l=0}^{+\infty}\left(1-\xi / \vartheta_{2 l+1}\right)}{\prod_{q=1}^{+\infty}\left(1-\xi / \vartheta_{2 q}\right)} .
$$

The expression of $D^{(1)}$ is unchanged but $D^{(2)}, D^{(3)}$ and $D^{(4)}$ are now defined as

$$
D^{(2)}\left(k_{x_{c}}\right)=-\sum_{l \geqslant 0} \frac{A_{2 l} \exp \left(\mathrm{i}\left(k_{x_{c}}-\delta_{2 l}\right) c\right) J_{-}\left(\delta_{2 l}\right)}{\mathrm{i}\left(\omega+k_{x_{c}} U\right)\left(k_{x_{c}}-\delta_{2 l}\right) J_{-}\left(k_{x_{c}}\right)},
$$


with

$$
\begin{aligned}
& A_{2 l}= \begin{cases}\frac{w_{0}\left(\omega+\delta_{2 l} U\right)}{(2 \pi)^{2}\left(\delta_{2 l}+k_{x_{c 0}}\right) J_{-}\left(-k_{x_{c 0}}\right) J_{+}^{\prime}\left(\delta_{2 l}\right)}, & \forall l \geqslant 1, \\
\frac{w_{0}\left(\omega-k_{x_{c 0}} U\right)}{(2 \pi)^{2} j\left(-k_{x_{c 0}}\right)}, & l=0,\end{cases} \\
& D^{(3)}=-\sum_{q=1}^{\infty} \frac{B_{2 q} J_{+}\left(\varepsilon_{2 q}\right)}{\left(k_{x_{c}}-\varepsilon_{2 q}\right) J_{+}\left(k_{x_{c}}\right)}, \\
& \left.D^{(4)}=-\sum_{p=0}^{\infty} \frac{C_{2 l} \exp \left(\mathrm{i}\left(k_{x_{c}}-\delta_{2 l}\right) c\right) J_{-}\left(\delta_{2 l}\right)}{\mathrm{i}\left(\omega+k_{x_{c}} U\right)\left(k_{x_{c}}-\delta_{2 l}\right) J_{-}\left(k_{x_{c}}\right)},\right\}
\end{aligned}
$$

where the coefficients $\boldsymbol{B}=\left(B_{2 q}\right)_{q \geqslant 1}$ and $\mathbf{C}=\left(C_{2 l}\right)_{l \geqslant 1}$ are solutions of the matrix system

$$
\left.\begin{array}{l}
\boldsymbol{B}=\boldsymbol{F} \cdot(\boldsymbol{A}+\boldsymbol{C}) \\
\boldsymbol{C}=\boldsymbol{L} \cdot \boldsymbol{B}
\end{array}\right\}
$$

with $\boldsymbol{A}=\left(A_{2 l}\right)_{l \geqslant 1}, \boldsymbol{F}=(F)_{(q, p) \in \mathbb{N}^{* 2}}, \boldsymbol{L}=(L)_{(q, p) \in \mathbb{N}^{* 2}}$ and

$$
F_{q, p}=-\frac{J_{-}\left(\delta_{2 l}\right) \exp \left(\mathrm{i}\left(\varepsilon_{2 q}-\delta_{2 l}\right) c\right)}{\mathrm{i}\left(\omega+\varepsilon_{2 q} U\right)\left(\varepsilon_{2 q}-\delta_{2 l}\right) J_{-}^{\prime}\left(\varepsilon_{2 q}\right)}, \quad L_{q, p}=\frac{\mathrm{i}\left(\omega+\delta_{2 q} U\right) J_{+}\left(\varepsilon_{2 l}\right)}{\left(\varepsilon_{2 l}-\delta_{2 q}\right) J_{+}^{\prime}\left(\delta_{2 q}\right)} .
$$

B.2. Acoustic potential in the inter-blade channel

The velocity potential is again given by

$$
\widehat{\phi}\left(x_{c}, y_{c}, z_{c}, t\right)=\int_{-\infty}^{\infty} D\left(k_{x_{c}}\right) A_{c}\left(k_{x_{c}}, y_{c}\right) \exp \left(-\mathrm{i} k_{x_{c}} x_{c}-\mathrm{i} \omega_{e x} t+\mathrm{i} k_{z_{c}} z_{c}\right) \mathrm{d} k_{x_{c}},
$$

but now

$$
\begin{aligned}
A_{c}\left(k_{x_{c}}, y_{c}\right) & =-\frac{1}{2} \sum_{n} \operatorname{sign}\left(n h-y_{c}\right) \exp \left(\mathrm{i} \zeta\left|n h-y_{c}\right|\right)=\frac{1}{2}\left[\frac{\mathrm{e}^{\mathrm{i} \zeta y_{c}}}{1-\mathrm{e}^{\mathrm{i} \zeta h}}+\frac{\mathrm{e}^{-\mathrm{i} \zeta y_{c}}}{1-\mathrm{e}^{-\mathrm{i} \zeta h}}\right] \\
& =-\frac{\cos \left(\zeta y_{c}\right)-\cos (\zeta(y-h))}{2[\cos (\zeta h)-1]}=-\frac{\sin \left(\zeta\left(y_{c}-h / 2\right)\right)}{2 \sin (\zeta h / 2)} .
\end{aligned}
$$

The acoustic potential inside the inter-blade channel (patch (b) only), for all $\left(x_{c}, y_{c}\right) \in$ $[0, c] \times \mathbb{R}$, is equal to

$$
\phi_{a c}\left(x_{c}, y_{c}\right)=\sum_{l \geqslant 1}\left\{\phi_{a c, b, 1, l}^{+}\left(x_{c}, y_{c}\right)+\sum_{q \geqslant 0}\left(\phi_{a c, b l, q, q}^{+}\left(x_{c}, y_{c}\right)+\phi_{a c, b, l, q}^{-}\left(x_{c}, y_{c}\right)\right)\right\},
$$

with

$$
\begin{aligned}
& \phi_{a c, b, l, q}^{-}\left(x_{c}, y_{c}\right)=\frac{8 \pi^{2}}{h} \frac{\left(A_{2 q}+C_{2 q}\right) \exp \left(\mathrm{i}\left(\varepsilon_{2 l}\left(c-x_{c}\right)-\delta_{2 q} c\right)\right) J_{-}\left(\delta_{2 q}\right) J_{+}\left(\varepsilon_{2 l}\right) \cos \left(\zeta\left(\varepsilon_{2 l}\right) y_{c}\right)}{\left(\omega+\varepsilon_{2 l} U\right)\left(\varepsilon_{2 l}-\delta_{2 l}\right) \zeta\left(\varepsilon_{2 q}\right)}, \\
& \phi_{a c, b, l, q}^{+}\left(x_{c}, y_{c}\right)=\frac{2(2 \pi)^{2} B_{2 q} J_{+}\left(\varepsilon_{2 q}\right) J_{-}\left(\delta_{2 l}\right) \cos \left(\zeta\left(\delta_{2 l}\right) y_{c}\right) \mathrm{e}^{-i \delta_{2 l} x_{c}}}{\left(\delta_{2 l}-\varepsilon_{2 q}\right) \zeta\left(\delta_{2 l}\right) h} \\
& \phi_{a c, b, 1, l}^{+}\left(x_{c}, y_{c}\right)=\frac{2 w_{0} J_{-}\left(\delta_{2 l}\right) \cos \left(\zeta\left(\delta_{2 l}\right) y_{c}\right) \mathrm{e}^{-\mathrm{i} \delta_{2 l} x_{c}}}{J_{-}\left(-k_{x_{c 0}}\right)\left(\delta_{2 l}+k_{x_{c 0}}\right) \zeta\left(\delta_{2 l}\right) h}
\end{aligned}
$$




\section{B.3. Pressure jump on the blade}

Finally, the expression for the pressure jump can then be derived from the acoustic potential and is written as

$$
\Delta p_{0}\left(x_{c}\right)=(D P 6+D P 8+D P 9)\left(x_{c}\right),
$$

with

$$
\left.\begin{array}{l}
D P 6\left(x_{c}\right)=\frac{16(\pi)^{2} \rho_{0} \mathrm{i}}{h} \sum_{l \geqslant 1} \sum_{q \geqslant 0} \frac{\left(A_{2 q}+C_{2 q}\right) J_{+}\left(\varepsilon_{2 l}\right) J_{-}\left(\delta_{2 q}\right) \exp \left(\mathrm{i}\left(\varepsilon_{2 l}\left(c-x_{c}\right)-\mathrm{i} \delta_{2 q} c\right)\right)}{\left(\varepsilon_{2 l}-\delta_{2 q}\right) \zeta\left(\varepsilon_{2 l}\right)}, \\
D P 8\left(x_{c}\right)=\rho_{0} \frac{4 \mathrm{i}}{h} \sum_{l \geqslant 1} \frac{w_{0}\left(\omega+\delta_{2 l} U\right) J_{-}\left(\delta_{2 l}\right)}{\left(\delta_{2 l}+k_{x_{c 0}}\right) J_{-}\left(-k_{x_{c 0}}\right) \zeta\left(\delta_{2 l}\right)} \mathrm{e}^{-\mathrm{i} \delta_{2 l} x_{c}}, \\
D P 9\left(x_{c}\right)=\rho_{0} \frac{4 \mathrm{i}(2 \pi)^{2}}{h} \sum_{l \geqslant 1} \sum_{q \geqslant 1} \frac{B_{2 q} J_{+}\left(\varepsilon_{2 q}\right) J_{-}\left(\delta_{2 l}\right)\left(\omega+\delta_{2 l} U\right)}{\left(\delta_{2 l}-\varepsilon_{2 q}\right) \zeta\left(\delta_{2 l}\right)} \mathrm{e}^{-\mathrm{i} \delta_{2 l} x_{c c}} .
\end{array}\right\}
$$

\section{REFERENCES}

Amiet, R. K. 1974 Compressibility effects in unsteady thin-airfoil theory. AIAA J. 12, 253-255.

AмIET, R. K. 1975 Effects of compressibility in unsteady airfoil lift theories. In University of Arizona/AFOSR Symposium on Unsteady Aerodynamics, Tucson, AZ (ed. R. B. Kinney). Arizona Board of Regents, Tucson.

Amiet, R. K. 1976 High frequency thin-airfoil theory for subsonic flow. AIAA J. 14, 1076-1082.

Atassi, H. M., Ali, A. A., Atassi, O. V. \& Vinogradov, I. V. 2004 Scattering of incidence disturbances by an annular cascade in a swirling flow. J. Fluid Mech. 499, 111-138.

Atassi, H. M. \& Hamad, G. 1981 Sound generated in a cascade by three-dimensional disturbances convected in subsonic flow. In 7th AIAA Aeroacoustics Conference, Palo Alto, CA, pp. 1-13.

Atassi, H. M. \& Logue, M. M. 2008 Effect of turbulence structure on broadband fan noise. In 14th AIAA/CEAS Aeroacoustics Conference and Exhibit, Vancouver, Canada, pp. 1-14.

Atassi, H. M. \& Vinogradov, I. V. 2005 A model for fan broadband interaction noise in nonuniform flow. In 11th AIAA/CEAS Aeroacoustics Conference and Exhibit, Monterey, CA, pp. 1-12.

Atassi, H. M. \& Vinogradov, I. V. 2007 Modelling broadband fan noise and comparison with experiments. In 13th AIAA/CEAS Aeroacoustics Conference and Exhibit, Rome, Italy, pp. $1-13$.

Boquilion, O., GlegG, S. A. L., Devenport, W. J. \& Larsan, J. 2003 The interaction of large scale turbulence with a cascade of flat plates. In 9th AIAA/CEAS Aeroacoustics Conference and Exhibit, Hilton Head, SC, pp. 1-8.

Cheong, C., Joseph, P. \& Soogab, L. 2006 High-frequency formulation for the acoustic power spectrum due to cascade-turbulence interaction. J. Acoust. Soc. Am. 119 (1), 108-122.

Elhadidi, B. \& Atassi, H. M. 2002 High frequency sound radiation from an annular cascade in swirling flows. In 8th AIAA/CEAS Aeroacoustics Conference and Exhibit, Breckenridge, CO, pp. 1-11.

Evers, I. \& Peake, N. 2002 On sound generation by the interaction between turbulence and a cascade of airfoils with non-uniform mean flow. J. Fluid Mech. 463, 25-52.

FANG, J. \& ATASSI, H. M. 1993 Compressible flows with vortical disturbances around a cascade of loaded airfoils. In Unsteady Aerodynamics, Aeroacoustics, and Aeroelasticity of Turbomachines and Propellers, pp. 149-176. Springer.

Ffowcs-Williams, J. E. \& Hawkings, D. L. 1969 Sound generation by turbulence and surfaces in arbitrary motion. Phil. Trans. R. Soc. Lond. A 264, 321-342.

Ganz, U. W., Joppa, P. D., Patten, T. J. \& Scharpf, D. F. 1998 Boeing 18-inch fan rig broadband noise test. Contractor Rep. CR-1998-208704. NASA.

GlegG, S. A. L. 1999 The response of a swept blade row to a three-dimensional gust. J. Sound Vib. 227 (1), 29-64.

Goldstein, M. E. 1976 Aeroacoustics. McGraw-Hill. 
Golubev, V. V. \& Atassi, H. M. 2000 Unsteady swirling flows in annular cascades, Part 2: Aerodynamic blade response incident disturbance. AIAA J. 38 (7), 1150-1158.

Graham, J. M. R. 1970 Similarity rules for thin aerofoils in non-stationary subsonic flows. J. Fluid Mech. 43 (4), 753-766.

Grissom, D. L., Devenport, W. J. \& GlegG, S. A. L. 2005 Measurements of a discrete upwash gust passing through a linear flat plate cascade, and comparisons with linearized theory. In 11th AIAA/CEAS Aeroacoustics Conference and Exhibit, Monterey, CA, pp. 1-23.

Hall, K. C. \& Verdon, J. M. 1991 Gust response analysis for cascades operating in nonuniform mean flows. AIAA J. 29 (9), 1463-1471.

Hanson, D. B. 1974 The spectrum of rotor noise caused by atmospheric turbulence. J. Acoust. Soc. Am. 56 (1), 110-126.

Hanson, D. B. 1994 Coupled 2-dimensional cascade theory for noise and unsteady aerodynamics of blade row interaction in turbofans. Vol. I. Theory development and parametric studies. Contractor Rep. CR-4506. NASA.

Hanson, D. B. 1999 Acoustic reflexion and transmission of 2-dimensional rotors and stators, including mode and frequency scattering effects. Contractor Rep. CR-1999-208880. NASA.

Hardin, J. C., Huff, D. \& TAM, C. K. 2000 Benchmark problems. In Third Computational Aeroacoustics (CAA) Workshop on Benchmark Problems (ed. M. D. Dahl), pp. 1-22. NASA Glenn Research Center.

KaJi, S. \& Okazaki, T. $1970 a$ Generation of sound by rotor-stator interaction. J. Sound Vib. 13, 281-307.

KaJI, S. \& OKaZaKi, T. $1970 b$ Propagation of sound waves through a blade row, II. Analysis based on the acceleration potential method. J. Sound Vib. 11 (3), 355-375.

von Kármán, Th. \& SeArs, W. R. 1938 Airfoil theory for nonuniform motion. J. Aero. Sci. 5 (10), 379-380.

Koch, W. 1971 On transmission of sound through a blade row. J. Sound Vib. 18 (1), 111-128.

Kodama, H. \& Namba, M. 1990 Unsteady lifting surface theory for a rotating cascade of swept blades. J. Turbomach. 112, 411-417.

Landahl, L. 1961 Unsteady Transonic Flow. Pergamon Press.

Lloyd, A. E. D. \& Peake, N. 2008 Rotor-stator broadband noise prediction. In 14th AIAA/CEAS Aeroacoustics Conference and Exhibit, Vancouver, Canada.

LockARD, D. P. 2000 An efficient, two-dimensional implementation of the Ffowcs Williams and Hawkings equation. J. Sound Vib. 229 (4), 897-911.

Majumdar, S. J. \& Peake, N. 1996 Three-dimensional effects in cascade-gust interaction. Wave Motion 23, 321-337.

Mani, R. \& Horvay, G. 1970 Sound transmission through blade rows. J. Sound Vib. 12 (1), 5983.

Namba, M. 1987 Three-dimensional flows. In Manual of Aeroelasticity in Axial Flow Turbomachinery (ed. M. F. Platzer \& F. O. Carta), vol. 1, Unsteady Turbomachinery Aerodynamics, chap. 4. Neuilly sur Seine, France: AGARD.

Namba, M. \& Schulten, J. B. H. M. 2000 Category 4 - fan stator with harmonic excitation by rotor wake. In Third Computational Aeroacoustics (CAA) Workshop on Benchmark Problems (ed. J. C. Hardin, D. Huff \& C. K. Tam), pp. 73-86.

Paterson, R. W. \& Amiet, R. K. 1979 Noise of a model helicopter rotor due to ingestion of turbulence. Contractor Rep. CR-3213. NASA.

Peake, N. 1992 The interaction between a high-frequency gust and a blade row. J. Fluid Mech. 241, 261-289.

PEAKE, N. 1993 The scattering of vorticity waves by an infinite cascade of flat plates in subsonic flow. Wave Motion 18, 255-271.

Peake, N. 2004 On application of high-frequency asymptotics in aeroacoustics. Phil. Trans. R. Soc. Lond. A 362, 673-696.

Peake, N. \& Kerschen, E. J. 1995 A uniform asymptotic approximation of high-frequency unsteady cascade flow. Proc. R. Soc. Lond. 449, 177-186.

Possio, C. 1938 L'azione aerodinamica sul profilo oscillante in un fluido compressible a velocita ipsonora. L'Aerotecnica 18 (4), 441-459.

Posson, H. 2008 Fonctions de réponse de grille d'aubes et effet d'écran pour le bruit à large bande des soufflantes. PhD thesis, Ecole Centrale de Lyon. 
Posson, H., Moreau, S. \& Roger, M. 2010 On the use of a uniformly valid analytical cascade response function for broadband noise predictions. J. Sound Vib. 329 (18), 3721-3743.

Posson, H. \& Roger, M. 2007 Parametric study of gust scattering and sound transmission through a blade row. In 13th AIAA/CEAS Aeroacoustics Conference and Exhibit, Rome, Italy, pp. 1-22.

Prasad, D. \& Verdon, J. M. 2002 A three-dimensional linearized Euler analysis of classical wake/stator interactions: validation and unsteady response predictions. Aeroacoustics 1 (2), $137-163$.

Ravindranath, A. \& Lakshminarayana, B. 1980 Three dimensional mean flow and turbulence characteristics of the near wake of the compressor rotor blade. Contractor Rep. 159518 PSU Turbo R 80-4. NASA.

REISSNER, E. 1951 On the application of mathieu functions in the theory of subsonic compressible flow past oscillating airfoils. Tech. Rep. TN 2363. NACA.

Schulten, J. B. H. M. 1982 Sound generated by rotor wakes interacting with a leaned vane stator. AIAA J. 20 (10), 1352-1358.

Schulten, J. B. H. M. 1997 Vane sweep effects on rotor/stator interaction noise. AIAA J. 35, 945-951.

SEARS, W. R. 1941 Some aspects of non-stationary airfoil theory and its practical applications. J. Aeronaut. Sci. 8 (3), 104-108.

Smith, S. N. 1973 Discrete frequency sound generation in axial flow turbomachines. Brit. Aero. Res. Counc. $R$ \&M 3709, 1-59.

Verdon, J. M. \& Hall, K. C. 1990 Development of a linearized unsteady aerodynamic analysis for cascade gust response predictions. Contractor Rep. CR-4308. NASA.

WhiteHEAD, D. S. 1972 Vibration and sound generation in a cascade of flat plates in subsonic flow. Tech. Rep. CUED/A-Turbo/TR 15. Cambridge University Engineering Laboratory.

Whitehead, D. S. 1987 Classical two-dimensional methods. In Manual of Aeroelasticity in Axial Flow Turbomachinery (ed. M. F. Platzer \& F. O. Carta), vol. 1, Unsteady Turbomachinery Aerodynamics, chap. 3. Neuilly sur Seine, France: AGARD. 